\title{
Volume and Self-Intersection of Differences of Two Nef Classes
}

\author{
Dan Popovici
}

\begin{abstract}
Let $\{\alpha\}$ and $\{\beta\}$ be nef cohomology classes of bidegree $(1,1)$ on a compact $n$-dimensional Kähler manifold $X$ such that the difference of intersection numbers $\{\alpha\}^{n}-n\{\alpha\}^{n-1} \cdot\{\beta\}$ is positive. We solve in a number of special but rather inclusive cases the quantitative part of Demailly's Transcendental Morse Inequalities Conjecture for this context predicting the lower bound $\{\alpha\}^{n}-n\{\alpha\}^{n-1}$. $\{\beta\}$ for the volume of the difference class $\{\alpha-\beta\}$. We completely solved the qualitative part in an earlier work. We also give general lower bounds for the volume of $\{\alpha-\beta\}$ and show that the self-intersection number $\{\alpha-\beta\}^{n}$ is always bounded below by $\{\alpha\}^{n}-n\{\alpha\}^{n-1} \cdot\{\beta\}$. We also describe and estimate the relative psef and nef thresholds of $\{\alpha\}$ with respect to $\{\beta\}$ and relate them to the volume of $\{\alpha-\beta\}$. Finally, broadening the scope beyond the Kähler realm, we propose a conjecture relating the balanced and the Gauduchon cones of $\partial \bar{\partial}$-manifolds which, if proved to hold, would imply the existence of a balanced metric on any $\partial \bar{\partial}$-manifold.
\end{abstract}

Mathematics Subject Classification (2010): 32J27, 32U40, 32Q25, 32J25, 53C55.

\section{Introduction}

Let $X$ be a compact Kähler manifold with $\operatorname{dim}_{\mathbb{C}} X=n$ and let $\{\alpha\},\{\beta\} \in H_{B C}^{1,1}(X, \mathbb{R})$ be nef Bott-Chern cohomology classes such that

$$
\{\alpha\}^{n}-n\{\alpha\}^{n-1} \cdot\{\beta\}>0 .
$$

A (possibly transcendental) class $\{\alpha\} \in H_{B C}^{1,1}(X, \mathbb{R})$ being nef means (cf. Definition 1.3 in [Dem92]) that for some (hence all) fixed Hermitian metric $\omega$ on $X$ and for every $\varepsilon>0$, there exists a $C^{\infty}$ form $\alpha_{\varepsilon} \in\{\alpha\}$ such that $\alpha_{\varepsilon} \geq-\varepsilon \omega$.

We have proved in [Pop14, Theorem 1.1] that the class $\{\alpha-\beta\}$ is big (i.e. contains a Kähler current T). This solved the qualitative part of Demailly's Transcendental Morse Inequalities Conjecture for differences of two nef classes (cf. [BDPP13, Conjecture 10.1, (ii)]) on compact Kähler (and even more general) manifolds. This special form of the conjecture was originally motivated by attempts at extending to transcendental classes and to compact Kähler (not necessarily projective) manifolds the cone duality theorem of Boucksom, Demailly, Paun and Peternell [BDPP13, Theorem 2.2.] that plays a major role in the theory of classification of projective manifolds. Recall that $T$ being a Kähler current means that $T$ is a $d$-closed positive $(1,1)$-current with the property that for some (hence all) fixed Hermitian metric $\omega$ on $X$, there exists $\varepsilon>0$ such that $T \geq \varepsilon \omega$ on $X$. Nefness and bigness are quite different positivity properties for real (possibly transcendental) (1, 1)-classes and the by-now standard definitions just recalled extend classical algebraic definitions for integral classes on projective manifolds.

In this paper we give a partial answer to the quantitative part of Demailly's Transcendental Morse Inequalities Conjecture for differences of two nef classes:

Conjecture 1.1 ([BDPP13, Conjecture 10.1, (ii)]) Let $\{\alpha\},\{\beta\} \in H_{B C}^{1,1}(X, \mathbb{R})$ be nef classes satisfying condition (1) on a compact Kähler manifold $X$ with $\operatorname{dim}_{\mathbb{C}} X=n$. Then 


$$
\operatorname{Vol}(\{\alpha-\beta\}) \geq\{\alpha\}^{n}-n\{\alpha\}^{n-1} \cdot\{\beta\} .
$$

This is stated for arbitrary (i.e. possibly non-Kähler) compact complex manifolds in [BDPP13], but the volume is currently only known to be meaningful when $X$ is of class $\mathcal{C}$, a case reducible to the Kähler case by modifications. Thus, we may assume without loss of generality that $X$ is Kähler.

Recall that the volume is a way of gauging the "amount" of positivity of a class $\{\gamma\} \in H_{B C}^{1,1}(X, \mathbb{R})$ when $X$ is Kähler (or merely of class $\mathcal{C}$ ) and was introduced in [Bou02, Definition 1.3] as

$$
\operatorname{Vol}(\{\gamma\}):=\sup _{T \in\{\gamma\}, T \geq 0} \int_{X} T_{a c}^{n}
$$

if $\{\gamma\}$ is pseudo-effective (psef), i.e. if $\{\gamma\}$ contains a positive $(1,1)$-current $T \geq 0$, where $T_{a c}$ denotes the absolutely continuous part of $T$ in the Lebesgue decomposition of its coefficients (which are complex measures when $T \geq 0$ ). If the class $\{\gamma\}$ is not psef, then its volume is set to be zero. It was proved in [Bou02, Theorem 1.2] that this volume (which is always a finite non-negative quantity thanks to the Kähler, or more generally class $\mathcal{C}$, assumption on $X$ ) coincides with the standard volume of a holomorphic line bundle $L$ if the class $\{\gamma\}$ is integral (i.e. the first Chern class of some $L)$. Moreover, the class $\{\gamma\}$ is big (i.e. contains a Kähler current) if and only if its volume is positive, by [Bou02, Theorem 4.7].

Thus, under the assumptions of Conjecture 1.1, the main result in [Pop14] ensures that Vol $(\{\alpha-$ $\beta\})>0$. In other words, $\{\alpha-\beta\}$ is positive in the big sense. The special case when $\{\beta\}=0$ had been proved in [DP04, Theorem 2.12] and had served there as the main ingredient in the proof of the numerical characterisation of the Kähler cone. (In particular, the proof of the more general statement in [Pop14] reproves in a much simpler way the main technical result in [DP04].) The thrust of Conjecture 1.1 is to estimate from below the "amount" of positivity of the class $\{\alpha-\beta\}$.

A first group of results that we obtain in the present paper can be summed up in the following positive answer to Conjecture 1.1 under an extra assumption. Recall that for nef classes $\{\gamma\}$, the volume equals the top self-intersection $\{\gamma\}^{n}$ (cf. [Bou02, Theorem 4.1]), but for arbitrary classes, any order may occur between these two quantities.

Theorem 1.2 Let $X$ be compact Kähler manifold with $\operatorname{dim}_{\mathbb{C}} X=n$ and let $\{\alpha\},\{\beta\} \in H_{B C}^{1,1}(X, \mathbb{R})$ be nef classes such that $\{\alpha\}^{n}-n\{\alpha\}^{n-1} .\{\beta\}>0$. Suppose, moreover, that

$$
\operatorname{Vol}(\{\alpha-\beta\}) \geq\{\alpha-\beta\}^{n} .
$$

Then $\operatorname{Vol}(\{\alpha-\beta\}) \geq\{\alpha\}^{n}-n\{\alpha\}^{n-1} \cdot\{\beta\}$.

Although there are examples when the volume of $\{\alpha-\beta\}$ is strictly less than the top selfintersection, the assumption (4), that we hope to be able to remove in future work, is satisfied in quite a number of cases, e.g. when the class $\{\alpha-\beta\}$ is nef (treated in section 2).

Actually, we prove in full generality in section 5 the analogue of Conjecture 1.1 for the top self-intersection number $\{\alpha-\beta\}^{n}$ in place of the volume of $\{\alpha-\beta\}$.

Theorem 1.3 Let $X$ be a compact Kähler manifold with $\operatorname{dim}_{\mathbb{C}} X=n$ and let $\{\alpha\},\{\beta\} \in H_{B C}^{1,1}(X, \mathbb{R})$ be nef classes such that $\{\alpha\}^{n}-n\{\alpha\}^{n-1} \cdot\{\beta\}>0$. Then

$$
\{\alpha-\beta\}^{n} \geq\{\alpha\}^{n}-n\{\alpha\}^{n-1} \cdot\{\beta\}
$$


It is clear that Theorem 1.2 follows immediately from Theorem 1.3. Since the nef cone is the closure of the Kähler cone, we may assume without loss of generality that the classes $\{\alpha\}$ and $\{\beta\}$ are actually Kähler. Corcerning the volume of $\{\alpha-\beta\}$ in the general case (i.e. without assumption (4)), we prove a lower bound that is weaker than the expected lower bound (2) in a way that depends explicitly on how far the class $\{\alpha-\beta\}$ is from being nef. The nefness defect of $\{\alpha-\beta\}$ is defined explicitly and investigated in relation to the volume in subsections $4.2,4.3$ and 4.4. We call it the nef threshold (a term that is already present in the literature) of $\{\alpha\}$ w.r.t. $\{\beta\}$ and discuss it together with the analogous psef threshold of $\{\alpha\}$ w.r.t. $\{\beta\}$ in section 4. In $\S .4 .4$, we prove the following general lower bound for the volume of $\{\alpha-\beta\}$.

Theorem 1.4 Let $X$ be compact Kähler manifold with $\operatorname{dim}_{\mathbb{C}} X=n$ and let $\{\alpha\},\{\beta\} \in H_{B C}^{1,1}(X, \mathbb{R})$ be Kähler classes such that $\{\alpha\}^{n}-n\{\alpha\}^{n-1} \cdot\{\beta\}>0$. Let $s_{0}:=N^{(\beta)}(\alpha)>0$ be the nef threshold of $\{\alpha\}$ w.r.t. $\{\beta\}$. Then:

(i) if $s_{0} \geq 1$, the class $\{\alpha-\beta\}$ is nef and the optimal volume estimate (2) holds;

(ii) if $s_{0}<1$, the class $\{\alpha-\beta\}$ is not nef and the next volume estimate holds:

$$
\operatorname{Vol}(\{\alpha-\beta\}) \geq\left(\{\alpha\}^{n}-n\{\alpha\}^{n-1} \cdot\{\beta\}\right)\left(\frac{\{\alpha\}^{n}-n\{\alpha\}^{n-1} \cdot\{\beta\}}{\{\alpha\}^{n}-n s_{0}\{\alpha\}^{n-1} \cdot\{\beta\}}\right)^{n-1} .
$$

A very special case of this result was also observed independently in [Tos15] using the technique introduced in [Pop14].

Taking our cue from the estimates we obtain in section 3 for the supremum of $t \geq 0$ such that the class $\{\alpha\}-t\{\beta\}$ is psef in the setting of Conjecture 1.1, we define the psef and the nef thresholds of $\{\alpha\}$ w.r.t. $\{\beta\}$ as functions $P^{(\beta)}, N^{(\beta)}: H_{B C}^{1,1}(X, \mathbb{R}) \rightarrow \mathbb{R}$ by

$$
\text { (i) } P^{(\beta)}(\alpha):=\inf \int_{X} \alpha \wedge \gamma^{n-1} \text { and }(i i) \quad N^{(\beta)}(\alpha):=\inf \int_{Y} \alpha \wedge \omega^{n-p-1},
$$

where in $(i)$ the infimum is taken over all the Gauduchon metrics $\gamma$ on $X$ normalised by

$$
[\beta]_{B C} \cdot\left[\gamma^{n-1}\right]_{A}=\int_{X} \beta \wedge \gamma^{n-1}=1
$$

while in $(i i)$ the infimum is taken over all $p=0,1, \ldots, n-1$, over all the irreducible analytic subsets $Y \subset X$ such that codim $Y=p$ and over all Kähler classes $\{\omega\}$ normalised by $\int_{Y} \beta \wedge \omega^{n-p-1}=1$. The class $\{\beta\}$ is supposed to be big in the case of $P^{(\beta)}$ and Kähler in the case of $N^{(\beta)}$. (The subscripts $\mathrm{BC}$ and A will stand throughout for the Bott-Chern, resp. Aeppli cohomologies.) In section 4, we prove the following formulae that justify the terminology and make it match existing notions in the literature:

$$
\begin{aligned}
& P^{(\beta)}(\alpha)=\sup \{t \in \mathbb{R} / \text { the class }\{\alpha\}-t\{\beta\} \text { is psef }\}, \\
& N^{(\beta)}(\alpha)=\sup \{s \in \mathbb{R} / \text { the class }\{\alpha\}-s\{\beta\} \text { is nef }\} .
\end{aligned}
$$

The psef/nef thresholds of $\{\alpha\}$ w.r.t. $\{\beta\}$ turn out to gauge quite effectively the amount of positivity that the class $\{\alpha\}$ has in the "direction" of the class $\{\beta\}$. We study their various properties in section 4 , estimate them in terms of intersection numbers as 


$$
\frac{\{\alpha\}^{n}}{n\{\alpha\}^{n-1} \cdot\{\beta\}} \leq P^{(\beta)}(\alpha) \leq \frac{\{\alpha\}^{n}}{\{\alpha\}^{n-1} \cdot\{\beta\}}
$$

and by similar, more involved inequalities for $N^{(\beta)}(\alpha)$, and relate them to the volume of $\{\alpha-\beta\}$ as

$$
\operatorname{Vol}(\{\alpha-\beta\}) \geq\left(1-\frac{1}{P^{(\beta)}(\alpha)}\right)^{n}\{\alpha\}^{n}
$$

whenever the classes $\{\alpha\}$ and $\{\beta\}$ are Kähler.

Using these thresholds, we prove Conjecture 1.1 in yet another special case: when the psef and the nef thresholds of $\{\alpha\}$ w.r.t. $\{\beta\}$ are sufficiently close to each other (cf. Proposition 4.12). Of course, we always have: $N^{(\beta)}(\alpha) \leq P^{(\beta)}(\alpha)$.

As in our earlier work [Pop14] and as in [Xia13] that preceded it, we will repeatedly make use of two ingredients. The first one is Lamari's positivity criterion.

Lemma 1.5 ([Lam99, Lemme 3.3]) Let $\{\alpha\} \in H_{B C}^{1,1}(X, \mathbb{R})$ be any real Bott-Chern cohomology class on an $n$-dimensional compact complex manifold $X$. The following two statements are equivalent.

(i) There exists a $(1,1)$-current $T$ in $\{\alpha\}$ such that $T \geq 0$ on $X$ (i.e. $\{\alpha\}$ is psef).

(ii) $\int_{X} \alpha \wedge \gamma^{n-1} \geq 0$ for all Gauduchon metrics $\gamma$ on $X$.

In fact, Lamari's result holds more generally for any (i.e. not necessarily $d$-closed) $C^{\infty}$ real $(1,1)$-form $\alpha$ on $X$, but we will not use this here. The second ingredient that we will often use is Yau's solution of the Calabi Conjecture.

Theorem 1.6 ([Yau78]) Let $X$ be a compact complex n-dimensional manifold endowed with a Kähler metric $\omega$. Let $d V>0$ be any $C^{\infty}$ positive volume form on $X$ such that $\int_{X} \omega^{n}=\int_{X} d V$. Then, there exists a unique Kähler metric $\widetilde{\omega}$ in the Kähler class $\{\omega\}$ such that $\widetilde{\omega}^{n}=d V$.

There is a non-Kähler analogue of Yau's theorem by Tosatti and Weinkove [TW10] that will not be used in this work. Moreover, most of the techniques that follow are still meaningful or can be extended to the non-Kähler context. This is part of the reason why we believe that a future development of the matters dealt with in this paper may be possible in the more general setting of $\partial \bar{\partial}$-manifolds. The conjecture we propose in section 6 is an apt illustration of this idea.

We will make repeated use of the technique based on the Cauchy-Schwarz inequality for estimating from below certain integrals of traces of Kähler metrics introduced in [Pop14]. Moreover, there are mainly two new techniques that we introduce in the current paper: $(i)$ the observation, proof and use of certain pointwise inequalities involving products of positive smooth forms (cf. Appendix) reminiscent of the Hovanskii-Teissier inequalities and generalising Lemma 3.1 in [Pop14]; (ii) a technique for constructing what we call approximate fixed points for Monge-Ampère equations when we allow the r.h.s. to vary (cf. proof of Proposition 5.1) whose rough idea originates in and was suggested by discussions the author had several years ago in a completely different context with different equations and for very different purposes with J.-P. Demailly to whom we are very grateful. 


\section{Special case of Conjecture 1.1 when $\{\alpha-\beta\}$ is nef}

We start by noticing the following elementary inequality.

Lemma 2.1 Let $\alpha>0$ and $\beta \geq 0$ be $C^{\infty}(1,1)$-forms on a complex manifold $X$ with dim $_{\mathbb{C}} X=n$ such that $\alpha-\beta \geq 0$. Then :

$$
(\alpha-\beta)^{n} \geq \alpha^{n}-n \alpha^{n-1} \wedge \beta \quad \text { at every point in } X .
$$

If $d \alpha=d \beta=0$ and if $X$ is compact, then taking integrals we get:

$$
\operatorname{Vol}(\{\alpha-\beta\})=\int_{X}(\alpha-\beta)^{n} \geq \int_{X} \alpha^{n}-n \int_{X} \alpha^{n-1} \wedge \beta=\{\alpha\}^{n}-n\{\alpha\}^{n-1} \cdot\{\beta\} .
$$

Proof. Let $x_{0} \in X$ be an arbitrary point and let $z_{1}, \ldots, z_{n}$ be local holomorphic coordinates centred at $x_{0}$ such that at $x_{0}$ we have:

$$
\alpha=\sum_{j=1}^{n} i d z_{j} \wedge d \bar{z}_{j} \quad \text { and } \quad \beta=\sum_{j=1}^{n} \beta_{j} i d z_{j} \wedge d \bar{z}_{j} .
$$

Then $\alpha-\beta=\sum_{j=1}^{n}\left(1-\beta_{j}\right) i d z_{j} \wedge d \bar{z}_{j}$ at $x_{0}$, while $\beta_{j} \geq 0$ and $1-\beta_{j} \geq 0$ at $x_{0}$ for all $j$. Thus inequality (6) at $x_{0}$ translates to

$$
\left(1-\beta_{1}\right) \cdots\left(1-\beta_{n}\right) \geq 1-\left(\beta_{1}+\cdots+\beta_{n}\right) \quad \text { for all } \beta_{1}, \ldots, \beta_{n} \in[0,1] .
$$

This elementary inequality is easily proved by induction on $n \geq 1$. Indeed, (8) is an identity for $n=1$, while if (8) has been proved for $n$, then we have:

$$
\begin{aligned}
\left(1-\beta_{1}\right) \cdots\left(1-\beta_{n}\right)\left(1-\beta_{n+1}\right) & \stackrel{(i)}{\geq}\left(1-\left(\beta_{1}+\cdots+\beta_{n}\right)\right)\left(1-\beta_{n+1}\right) \\
& =1-\left(\beta_{1}+\cdots+\beta_{n}+\beta_{n+1}\right)+\beta_{n+1}\left(\beta_{1}+\cdots+\beta_{n}\right) \\
& \geq 1-\left(\beta_{1}+\cdots+\beta_{n}+\beta_{n+1}\right)
\end{aligned}
$$

since $\beta_{j} \geq 0$ for all $j$. (We used $1-\beta_{n+1} \geq 0$ to get $(i)$ from the induction hypothesis.) Thus (8) is proved and (6) follows from it.

Now, if $\alpha$ and $\beta$ are $d$-closed, they define Bott-Chern cohomology classes. Since $\alpha-\beta$ is a semipositive $C^{\infty}(1,1)$-form, its Bott-Chern class is nef (and even a bit more), hence its volume equals $\int_{X}(\alpha-\beta)^{n}$ by [Bou02, Theorem 4.1] if $X$ is compact. (Note that $X$ is compact Kähler since $\alpha$ is a Kähler metric under the present assumptions.) The remaining part of (7) follows at once from (6) by integration.

An immediate consequence of Lemma 2.1 is the desired volume lower bound (2) in the special case when the class $\{\alpha-\beta\}$ is assumed to be nef. Note, however, that $\{\alpha-\beta\}$ need not be nef in general even a posteriori in the setting of Conjecture 1.1. 
Proposition 2.2 Let $X$ be a compact Kähler manifold with $\operatorname{dim}_{\mathbb{C}} X=n$ and let $\{\alpha\},\{\beta\} \in H_{B C}^{1,1}(X, \mathbb{R})$ be nef Bott-Chern cohomology classes such that the class $\{\alpha-\beta\}$ is nef. Then

$$
\operatorname{Vol}(\{\alpha-\beta\}) \geq\{\alpha\}^{n}-n\{\alpha\}^{n-1} \cdot\{\beta\} .
$$

Proof. It suffices to prove inequality (9) in the case when the classes $\{\alpha\},\{\beta\}$ and $\{\alpha-\beta\}$ are all Kähler. (Otherwise, we can add $2 \varepsilon\{\omega\}$ to $\{\alpha\}$ and $\varepsilon\{\omega\}$ to $\{\beta\}$ for a fixed Kähler class $\{\omega\}$ and let $\varepsilon \downarrow 0$ in the end. The volume function is known to be continuous by [Bou02, Corollary 4.11].) If we define the form $\alpha$ as the sum of any Kähler metric in the class $\{\alpha-\beta\}$ with any Kähler metric $\beta$ in the class $\{\beta\}$, the forms $\alpha, \beta$ and $\alpha-\beta$ obtained in this way satisfy the hypotheses of Lemma 2.1, hence also the elementary inequality (6) and its consequence (7).

Recall that the class $\{\alpha-\beta\}$ is big under the assumptions of Conjecture 1.1 by the main result in [Pop14]. However, big positivity is quite different in nature to nef positivity. The general (i.e. possibly non-nef) case is discussed in the next sections.

\section{Applications of Monge-Ampère equations}

In this section, we rewrite in a more effective way and observe certain consequences of the arguments in $[$ Pop14, §.3].

Lemma 3.1 Let $X$ be any compact complex manifold with $\operatorname{dim}_{\mathbb{C}} X=n$. With any $C^{\infty}(1,1)$-forms $\alpha, \beta>0$ and any Gauduchon metric $\gamma$, we associate the $C^{\infty}(1,1)$-form $\widetilde{\alpha}=\alpha+i \partial \bar{\partial} u>0$ defined as the unique normalised solution (whose existence is guaranteed by the Tosatti-Weinkove theorem in [TW10]) of the Monge-Ampère equation:

$$
(\alpha+i \partial \bar{\partial} u)^{n}=c \beta \wedge \gamma^{n-1} \quad \text { such that } \sup _{X} u=0
$$

where $c>0$ is the unique constant for which the above equation admits a solution $u: X \rightarrow \mathbb{R}$. (Of course, a posteriori, $c=\left(\int_{X}(\alpha+i \partial \bar{\partial} u)^{n}\right) /\left(\int_{X} \beta \wedge \gamma^{n-1}\right)$, while if $d \alpha=0$ then $c=\int_{X} \alpha^{n}=\{\alpha\}^{n}>0$.)

Then the following inequality holds:

$$
\left(\int_{X} \widetilde{\alpha} \wedge \gamma^{n-1}\right) \cdot\left(\int_{X} \widetilde{\alpha}^{n-1} \wedge \beta\right) \geq \frac{1}{n}\left(\int_{X} \widetilde{\alpha}^{n}\right)\left(\int_{X} \beta \wedge \gamma^{n-1}\right)
$$

Proof. Let us define $\operatorname{det}_{\gamma} \widetilde{\alpha}$ by requiring $\widetilde{\alpha}^{n}=\left(\operatorname{det}_{\gamma} \widetilde{\alpha}\right) \gamma^{n}$ on $X$. Since $\beta \wedge \gamma^{n-1}=(1 / n)\left(\Lambda_{\gamma} \beta\right) \gamma^{n}$, the Monge-Ampére equation (10) translates to

$$
\operatorname{det}_{\gamma} \widetilde{\alpha}=\frac{c}{n} \Lambda_{\gamma} \beta
$$

Hence, we get the following identities and inequalities:

$$
\left(\int_{X} \widetilde{\alpha} \wedge \gamma^{n-1}\right)\left(\int_{X} \widetilde{\alpha}^{n-1} \wedge \beta\right)=\left(\int_{X} \frac{1}{n}\left(\Lambda_{\gamma} \widetilde{\alpha}\right) \gamma^{n}\right)\left(\int_{X} \frac{1}{n}\left(\Lambda_{\widetilde{\alpha}} \beta\right)\left(\operatorname{det}_{\gamma} \widetilde{\alpha}\right) \gamma^{n}\right)
$$




$$
\begin{aligned}
\stackrel{(a)}{\geq} \frac{1}{n^{2}}\left(\int_{X}\left[\left(\Lambda_{\gamma} \widetilde{\alpha}\right)\left(\Lambda_{\widetilde{\alpha}} \beta\right)\right]^{\frac{1}{2}}\left(\operatorname{det}_{\gamma} \widetilde{\alpha}\right)^{\frac{1}{2}} \gamma^{n}\right)^{2} & \stackrel{(b)}{\geq} \frac{1}{n^{2}}\left(\int_{X}\left(\Lambda_{\gamma} \beta\right)^{\frac{1}{2}}\left(\operatorname{det}_{\gamma} \widetilde{\alpha}\right)^{\frac{1}{2}} \gamma^{n}\right)^{2} \\
\stackrel{(c)}{=} \frac{1}{n^{2}}\left(\sqrt{\frac{c}{n}} \int_{X}\left(\Lambda_{\gamma} \beta\right) \gamma^{n}\right)^{2} & =\frac{1}{n^{2}}\left(\sqrt{\frac{c}{n}} n \int_{X} \beta \wedge \gamma^{n-1}\right)^{2}=\frac{c}{n}\left(\int_{X} \beta \wedge \gamma^{n-1}\right)^{2},
\end{aligned}
$$

which prove (11) since $c=\left(\int_{X} \widetilde{\alpha}^{n}\right) /\left(\int_{X} \beta \wedge \gamma^{n-1}\right)>0$, where $(a)$ is the Cauchy-Schwarz inequality, (b) follows from the inequality $\left(\Lambda_{\gamma} \widetilde{\alpha}\right)\left(\Lambda_{\widetilde{\alpha}} \beta\right) \geq \Lambda_{\gamma} \beta$ proved in [Pop14, Lemma 3.1], while $(c)$ follows from (12).

Corollary 3.2 Let $X$ be a compact Kähler manifold with dim $_{\mathbb{C}} X=n$. Then, for every Kähler metrics $\alpha, \beta$ and every Gauduchon metric $\gamma$ on $X$, the following inequality holds:

$$
\left(\int_{X} \alpha \wedge \gamma^{n-1}\right) \cdot\left(\int_{X} \alpha^{n-1} \wedge \beta\right) \geq \frac{1}{n}\left(\int_{X} \alpha^{n}\right)\left(\int_{X} \beta \wedge \gamma^{n-1}\right) .
$$

Proof. It is clear that (13) follows immediately from (11) since the assumption $d \alpha=d \beta=0$ ensures that $\int_{X} \widetilde{\alpha} \wedge \gamma^{n-1}=\int_{X} \alpha \wedge \gamma^{n-1}, \int_{X} \widetilde{\alpha}^{n-1} \wedge \beta=\int_{X} \alpha^{n-1} \wedge \beta$ and $\int_{X} \widetilde{\alpha}^{n}=\int_{X} \alpha^{n}$.

Remark 3.3 Under the hypotheses of Corollary 3.2, for any $\gamma$ satisfying the inequality $\left(\Lambda_{\gamma} \widetilde{\alpha}\right)\left(\Lambda_{\widetilde{\alpha}} \beta\right) \geq$ $n \Lambda_{\gamma} \beta$ (an improved version of [Pop14, Lemma 3.1] which need not hold in general, but holds for some special choices of $\gamma-c f$. proof of Lemma 7.2), the lower bound on the r.h.s. of (13) improves to $\left(\int_{X} \alpha^{n}\right)\left(\int_{X} \beta \wedge \gamma^{n-1}\right)$. If this improved lower bound held for all Gauduchon metrics $\gamma$, Conjecture 1.1 would follow immediately (see Theorem 3.5 below).

We first notice a consequence of Corollary 3.2 for nef classes.

Corollary 3.4 If $\{\alpha\}$ and $\{\beta\} \in H_{B C}^{1,1}(X, \mathbb{R})$ are nef classes on a compact Kähler manifold $X$ with $\operatorname{dim}_{\mathbb{C}} X=n$ such that $\{\alpha\}^{n}-n\{\alpha\}^{n-1} \cdot\{\beta\}>0$, then $\{\alpha\}^{n}>0$ and, unless $\{\beta\}=0$, the following non-orthogonality property holds: $\{\alpha\}^{n-1} \cdot\{\beta\}>0$.

Proof. The nef hypothesis on $\{\alpha\}$ and $\{\beta\}$ ensures that $\{\alpha\}^{n-1} \cdot\{\beta\} \geq 0$, hence $\{\alpha\}^{n}>0$ since $\{\alpha\}^{n}>n\{\alpha\}^{n-1} .\{\beta\}$ by assumption. For the rest of the proof, we reason by contradiction: suppose that $\{\alpha\}^{n-1} \cdot\{\beta\}=0$ and that $\{\beta\} \neq 0$. By the nef hypothesis on $\{\alpha\}$ and $\{\beta\}$, for every $\varepsilon>0$, there exist $C^{\infty}$ forms $\alpha_{\varepsilon} \in\{\alpha\}, \beta_{\varepsilon} \in\{\beta\}$ such that $\alpha_{\varepsilon}+\varepsilon \omega>0$ and $\beta_{\varepsilon}+\varepsilon \omega>0$ for some arbitrary fixed Kähler metric $\omega$ on $X$. Applying (13) to the Kähler metrics $\alpha_{\varepsilon}+\varepsilon \omega$ and $\beta_{\varepsilon}+\varepsilon \omega$ in place of $\alpha$ and $\beta$ and letting $\varepsilon \downarrow 0$, we get $\int_{X} \beta \wedge \gamma^{n-1}=0$ for every Gauduchon metric $\gamma$ on $X$. (Note that $\int_{X} \alpha_{\varepsilon} \wedge \gamma^{n-1}=\int_{X} \alpha \wedge \gamma^{n-1}, \int_{X} \beta_{\varepsilon} \wedge \gamma^{n-1}=\int_{X} \beta \wedge \gamma^{n-1}$ and $\int_{X} \alpha_{\varepsilon}^{n-1} \wedge \beta_{\varepsilon}=\{\alpha\}^{n-1} .\{\beta\}=0$.) If we fix a $d$-closed positive current $T \geq 0$ in the class $\{\beta\}$ (such a current exists since the nef class $\{\beta\}$ is, in particular, pseudo-effective), this means that $\int_{X} T \wedge \gamma^{n-1}=0$ for every Gauduchon metric $\gamma$ on $X$. Consequently, $T=0$, hence $\{\beta\}=\{T\}=0$, a contradiction.

An immediate consequence of Corollary 3.2 is the following result in which the volume lower bound (15) falls short of the expected inequality (2). However, (15) solves the qualitative part of [BDPP13, Conjecture 10.1, (ii)] already solved in [Pop14], while (14) gives moreover an effective estimate of the largest $t>0$ for which the class $\{\alpha-t \beta\}$ remains pseudo-effective. This estimate will prompt the discussion of the psef and nef thresholds in the next section. 
Theorem 3.5 Let $X$ be a compact Kähler manifold with $\operatorname{dim}_{\mathbb{C}} X=n$ and let $\alpha, \beta>0$ be Kähler metrics such that $\{\alpha\}^{n}-n\{\alpha\}^{n-1} .\{\beta\}>0$.

Then, for every $t \in[0,+\infty)$, there exists a real $(1,1)$-current $T_{t} \in\{\alpha-t \beta\}$ such that

$$
T_{t} \geq\left(1-n t \frac{\{\alpha\}^{n-1} \cdot\{\beta\}}{\{\alpha\}^{n}}\right) \alpha \text { on } X .
$$

In particular, $T_{t}$ is a Kähler current for all $0 \leq t<\frac{\{\alpha\}^{n}}{n\{\alpha\}^{n-1} \cdot\{\beta\}}$, so taking $t=1$ (which is allowed by the assumption $\left.\{\alpha\}^{n}-n\{\alpha\}^{n-1} .\{\beta\}>0\right)$ we get that the class $\{\alpha-\beta\}$ contains a Kähler current. Moreover, its volume satisfies:

$$
\operatorname{Vol}(\{\alpha-\beta\}) \geq\left(\{\alpha\}^{n}-n\{\alpha\}^{n-1} \cdot\{\beta\}\right)\left(\frac{\{\alpha\}^{n}-n\{\alpha\}^{n-1} \cdot\{\beta\}}{\{\alpha\}^{n}}\right)^{n-1} \geq\{\alpha\}^{n}-n^{2}\{\alpha\}^{n-1} \cdot\{\beta\} .
$$

Proof. Thanks to Lamari's positivity criterion (Lemma 1.5), the existence of a current $T_{t} \in\{\alpha-t \beta\}$ satisfying (14) is equivalent to

$$
\int_{X}\left(\alpha-t \beta-\alpha+n t \frac{\{\alpha\}^{n-1} \cdot\{\beta\}}{\{\alpha\}^{n}} \alpha\right) \wedge \gamma^{n-1} \geq 0
$$

for every Gauduchon metric $\gamma$ on $X$. This, in turn, is equivalent to

$$
n t \frac{\{\alpha\}^{n-1} \cdot\{\beta\}}{\{\alpha\}^{n}} \int_{X} \alpha \wedge \gamma^{n-1} \geq t \int_{X} \beta \wedge \gamma^{n-1} \text { for every Gauduchon metric } \gamma .
$$

The last inequality is nothing but (13) which was proved in Corollary 3.2. This completes the proof of the existence of a current $T_{t} \in\{\alpha-t \beta\}$ satisfying (14).

Now, (14) implies that the absolutely continuous part $T_{a c}$ of $T:=T_{1} \in\{\alpha-\beta\}$ has the same lower bound as $T$. Moreover, if $\{\alpha\}^{n}-n\{\alpha\}^{n-1} .\{\beta\}>0$, then

$$
\operatorname{Vol}(\{\alpha-\beta\}) \geq \int_{X} T_{a c}^{n} \geq\left(1-n \frac{\{\alpha\}^{n-1} \cdot\{\beta\}}{\{\alpha\}^{n}}\right)^{n} \int_{X} \alpha^{n} \stackrel{(i)}{\geq}\left(1-n^{2} \frac{\{\alpha\}^{n-1} \cdot\{\beta\}}{\{\alpha\}^{n}}\right)\{\alpha\}^{n},
$$

which proves the claim (15). To obtain $(i)$, we have used the elementary inequality $(1-\lambda)^{n} \geq 1-n \lambda$ which holds for every $\lambda \in[0,1]$.

The above proof shows that a current $T_{t} \in\{\alpha-t \beta\}$ satisfying (14) exists even if we do not assume $\{\alpha\}^{n}-n\{\alpha\}^{n-1} \cdot\{\beta\}>0$, although this information will be of use only under this assumption. Note that the non-orthogonality property $\{\alpha\}^{n-1} \cdot\{\beta\}>0$ ensured by Corollary 3.4 constitutes the obstruction to the volume lower estimate (15) being optimal (i.e. coinciding with the expected estimate (2)). We now point out an alternative way of inferring the same suboptimal volume lower bound (15) from the proof of Theorem 3.5.

Alternative wording of the proof of the volume lower estimate (15). By Lamari's positivity criterion (Lemma 1.5), the existence of a current $T$ in the class $\{\alpha-\beta\}$ such that $T \geq \delta \alpha$ for some constant $\delta>0$ (which must be such that $\delta<1$ ) is equivalent to 


$$
\int_{X}((1-\delta) \alpha-\beta) \wedge \gamma^{n-1} \geq 0, \quad \text { i.e. to } \int_{X}\left(\left(\alpha-\frac{1}{1-\delta} \beta\right) \wedge \gamma^{n-1} \geq 0,\right.
$$

for all Gauduchon metrics $\gamma$ on $X$. Applying again Lamari's positivity criterion, this is still equivalent to the class $\{\alpha\}-(1 /(1-\delta))\{\beta\}$ being pseudo-effective. Inequality (14) shows that the largest $\delta$ we can choose with this property is larger than or equal to

$$
\delta_{0}=1-n \frac{\{\alpha\}^{n-1} \cdot\{\beta\}}{\{\alpha\}^{n}}, \quad \text { which gives } 1-\delta_{0}=n \frac{\{\alpha\}^{n-1} \cdot\{\beta\}}{\{\alpha\}^{n}} .
$$

On the other hand, we can write:

$$
\operatorname{Vol}(\{\alpha-\beta\})=\operatorname{Vol}\left((1-t)\{\alpha\}+t\left(\{\alpha\}-\frac{1}{t}\{\beta\}\right)\right) \stackrel{(a)}{\geq} \operatorname{Vol}((1-t)\{\alpha\})=(1-t)^{n}\{\alpha\}^{n}
$$

where inequality $(a)$ holds for every $t \in[0,1]$ for which the class $\{\alpha\}-(1 / t)\{\beta\}$ is pseudo-effective. By (16), $t:=1-\delta_{0}=n\{\alpha\}^{n-1} \cdot\{\beta\} /\{\alpha\}^{n}$ satisfies this property. With this choice of $t$, inequality (17) translates to the first inequality in (15).

Corollary 3.6 Let $\{\alpha\},\{\beta\} \in H_{B C}^{1,1}(X, \mathbb{R})$ be nef classes on a compact Kähler manifold $X$ with $\operatorname{dim}_{\mathbb{C}} X=n$ such that $\{\alpha\}^{n}-n\{\alpha\}^{n-1} \cdot\{\beta\}>0$. If $\{\beta\}=0$, then $\{\alpha\}$ is big, while if $\{\beta\} \neq 0$, then $\{\alpha\}-t\{\beta\}$ is big for all $0 \leq t<\frac{\{\alpha\}^{n}}{n\{\alpha\}^{n-1} \cdot\{\beta\}}$. Moreover, the volume lower bound (15) holds.

The case when $\{\beta\}=0$ is the key Theorem 2.12 in [DP04]. So, in particular, our method produces a much quicker proof of this fundamental result of [DP04]. The case when $\{\beta\} \neq 0$ is new, although the case $t=1$ and the method of proof are those of [Pop14]. Notice that the quantity $\{\alpha\}^{n} / n\{\alpha\}^{n-1} .\{\beta\}>0$ is well defined when $\{\beta\} \neq 0$ by Corollary 3.4.

Proof. We fix an arbitrary Kähler metric $\omega$ on $X$ and a constant $t \geq 0$ that will be specified shortly. The nefness assumption on $\{\alpha\},\{\beta\}$ means that for every $\varepsilon>0$, smooth forms $\alpha \in\{\alpha\}$ and $\beta \in\{\beta\}$ depending on $\varepsilon$ can be found such that $\alpha_{\varepsilon}:=\alpha+\varepsilon \omega$ and $\beta_{\varepsilon}:=\beta+\frac{\varepsilon}{t} \omega$ are Kähler metrics. Notice that the class $\left\{\alpha_{\varepsilon}-t \beta_{\varepsilon}\right\}=\{\alpha-t \beta\}$ is independent of $\varepsilon$. On the other hand, the quantities $\left\{\alpha_{\varepsilon}\right\}^{n}=$ $\{\alpha\}^{n}+\sum_{k=1}^{n} \varepsilon^{k}\left(\begin{array}{l}n \\ k\end{array}\right)\{\alpha\}^{n-k} \cdot\{\omega\}^{k}$ and $\left\{\alpha_{\varepsilon}\right\}^{n-1} \cdot\left\{\beta_{\varepsilon}\right\}=\left(\{\alpha\}^{n-1}+\sum_{l=1}^{n-1} \varepsilon^{l}\left(\begin{array}{c}n-1 \\ l\end{array}\right)\{\alpha\}^{n-1-l} \cdot\{\omega\}^{l}\right) \cdot\left(\{\beta\}+\frac{\varepsilon}{t}\{\omega\}\right)$ converge to $\{\alpha\}^{n}$ and respectively $\{\alpha\}^{n-1} .\{\beta\}$ when $\varepsilon \rightarrow 0$. Thus, $\left\{\alpha_{\varepsilon}\right\}^{n}-n\left\{\alpha_{\varepsilon}\right\}^{n-1} .\left\{\beta_{\varepsilon}\right\}>0$ if $\varepsilon>0$ is small enough. Applying Theorem 3.5 to the Kähler metrics $\alpha_{\varepsilon}$ and $\beta_{\varepsilon}$, we infer that the class $\left\{\alpha_{\varepsilon}-t \beta_{\varepsilon}\right\}=\{\alpha-t \beta\}$ is big whenever $0 \leq t<\left\{\alpha_{\varepsilon}\right\}^{n} / n\left\{\alpha_{\varepsilon}\right\}^{n-1} \cdot\left\{\beta_{\varepsilon}\right\}$. In particular, if $\{\beta\}=0$, this means that the class $\{\alpha\}$ is big (since we can fix $\varepsilon>0$ and choose $t=0$ ). Meanwhile, if $\{\beta\} \neq 0$ and if we choose $t<\{\alpha\}^{n} / n\{\alpha\}^{n-1} \cdot\{\beta\}$, then $t<\left\{\alpha_{\varepsilon}\right\}^{n} / n\left\{\alpha_{\varepsilon}\right\}^{n-1} \cdot\left\{\beta_{\varepsilon}\right\}$ for all $\varepsilon>0$ small enough and we conclude that $\{\alpha-t \beta\}$ is big. The volume lower bound (15) holds for $\left\{\alpha_{\varepsilon}\right\}$ and $\left\{\beta_{\varepsilon}\right\}$ for $t=1$ and all sufficiently small $\varepsilon>0$, so letting $\varepsilon \rightarrow 0$ and using the continuity of the volume, we get it for $\{\alpha\}$ and $\{\beta\}$. 


\section{Trace and volume of $(1,1)$-cohomology classes}

The implicit discussion of the relative positivity thresholds of a cohomology class with respect to another in Theorem 3.5 and in Corollary 3.6 prompts a further investigation of their relationships with the volume that we undertake to study in this section.

\subsection{The psef threshold}

Let $X$ be a compact complex manifold in Fujiki's class $\mathcal{C}, n:=\operatorname{dim}_{\mathbb{C}} X$.

Definition 4.1 For every big Bott-Chern class $\{\beta\}=[\beta]_{B C} \in H_{B C}^{1,1}(X, \mathbb{R})$, we define the $\beta$ directed trace (or the psef threshold in the $\beta$-direction) to be the function:

$$
P^{(\beta)}: H_{B C}^{1,1}(X, \mathbb{R}) \rightarrow \mathbb{R}, \quad P^{(\beta)}(\alpha):=\inf \int_{X} \alpha \wedge \gamma^{n-1},
$$

for all Bott-Chern classes $\{\alpha\}=[\alpha]_{B C} \in H_{B C}^{1,1}(X, \mathbb{R})$, where the infimum is taken over all the Gauduchon metrics $\gamma$ on $X$ normalised such that

$$
[\beta]_{B C} \cdot\left[\gamma^{n-1}\right]_{A}=\int_{X} \beta \wedge \gamma^{n-1}=1
$$

All the integrals involved in the above definition are clearly independent of the representatives $\alpha, \beta$ of the Bott-Chern classes $[\alpha]_{B C},[\beta]_{B C}$ and of the representative $\gamma^{n-1}$ of the Aeppli-Gauduchon class $\left[\gamma^{n-1}\right]_{A} \in H_{A}^{n-1, n-1}(X, \mathbb{R})$. Thus the infimum is taken over the subset $S_{\beta}$ of the Gauduchon cone $\mathcal{G}_{X}$ consisting of classes $\left[\gamma^{n-1}\right]_{A}$ normalised by $[\beta]_{B C} \cdot\left[\gamma^{n-1}\right]_{A}=1$. The bigness assumption on $[\beta]_{B C}$ has been imposed to ensure that $[\beta]_{B C} \cdot\left[\gamma^{n-1}\right]_{A}>0$, hence that $\left[\gamma^{n-1}\right]_{A}$ can be normalised w.r.t. $[\beta]_{B C}$ as in (19), for every class $\left[\gamma^{n-1}\right]_{A} \in \mathcal{G}_{X}$.

This definition is motivated in part by the next observation which is an immediate consequence of Lamari's positivity criterion: the $\beta$-directed trace $P^{(\beta)}$ coincides with the slope function introduced for big classes $\{\alpha\}$ in [BFJ09, Definition 3.7] and thus gauges the positivity of real $(1,1)$-classes $\{\alpha\}$ w.r.t. a reference big class $\{\beta\}$. The quantity on the r.h.s. of (20) below (i.e. the slope) may well be called the psef threshold of $\{\alpha\}$ in the $\{\beta\}$-direction (a term already used in the literature).

Proposition 4.2 Suppose that $\{\beta\} \in H_{B C}^{1,1}(X, \mathbb{R})$ is a fixed big class. Then

$$
\begin{aligned}
P^{(\beta)}(\alpha) & =\sup \{t \in \mathbb{R} / \text { the class }\{\alpha\}-t\{\beta\} \text { is psef }\} \\
& =\sup \left\{t \in \mathbb{R} / \exists T \in\{\alpha\} \text { current, } \exists \widetilde{\beta} \in\{\beta\} C^{\infty} \text {-form s.t. } T \geq t \widetilde{\beta}\right\} \\
& =\sup \left\{t \in \mathbb{R} / \forall \widetilde{\beta} \in\{\beta\} C^{\infty} \text {-form, } \exists T \in\{\alpha\} \text { current s.t. } T \geq t \widetilde{\beta}\right\},
\end{aligned}
$$

for every class $\{\alpha\} \in H_{B C}^{1,1}(X, \mathbb{R})$. In particular, the set $\{t \in \mathbb{R} /$ the class $\{\alpha\}-t\{\beta\}$ is psef $\}$ equals the interval $\left(-\infty, P^{(\beta)}(\alpha)\right]$. 
Proof. Let $A_{\alpha}^{\beta}:=\{t \in \mathbb{R} /$ the class $\{\alpha\}-t\{\beta\}$ is psef $\}$ and let $t_{\alpha}^{\beta}:=\sup A_{\alpha}^{\beta}$. By Lamari's positivity criterion, the class $\{\alpha\}-t\{\beta\}$ is psef iff

$$
\int_{X} \alpha \wedge \gamma^{n-1} \geq t \int_{X} \beta \wedge \gamma^{n-1} \quad \text { for all }\left[\gamma^{n-1}\right]_{A} \in \mathcal{G}_{X} \Longleftrightarrow \int_{X} \alpha \wedge \gamma^{n-1} \geq t
$$

for all $\left[\gamma^{n-1}\right]_{A} \in \mathcal{G}_{X}$ normalised such that $\int_{X} \beta \wedge \gamma^{n-1}=1$. This proves the inequality $P^{(\beta)}(\alpha) \geq t_{\alpha}^{\beta}$. To prove that equality holds, we reason by contradiction. Suppose that $P^{(\beta)}(\alpha)>t_{\alpha}^{\beta}$. Pick any $t_{1}$ such that $P^{(\beta)}(\alpha)>t_{1}>t_{\alpha}^{\beta}$. Then $\int_{X} \alpha \wedge \gamma^{n-1}>t_{1}$ for all Gauduchon metrics $\gamma$ such that $[\beta]_{B C} \cdot\left[\gamma^{n-1}\right]_{A}=1$. This is equivalent to $\int_{X}^{X} \alpha \wedge \gamma^{n-1}>t_{1} \int_{X} \beta \wedge \gamma^{n-1}$ for all Gauduchon metrics $\gamma$, which thanks to Lamari's positivity criterion implies:

$\exists T \in\{\alpha\}-t_{1}\{\beta\}$ such that $T \geq 0$, i.e. the class $\{\alpha\}-t_{1}\{\beta\}$ is psef.

Thus $t_{1} \in A_{\alpha}^{\beta}$, contradicting the choice $t_{1}>t_{\alpha}^{\beta}=\sup A_{\alpha}^{\beta}$.

An immediate consequence is the next statement showing that the $\beta$-directed trace $(=$ the psef threshold) gauges the positivity of real Bott-Chern $(1,1)$-classes much as the volume does.

Corollary $4.3(i)$ Suppose that $\{\beta\} \in H_{B C}^{1,1}(X, \mathbb{R})$ is a fixed big class. For any class $\{\alpha\} \in$ $H_{B C}^{1,1}(X, \mathbb{R})$, the following equivalences hold:

(i) $\{\alpha\}$ is psef $\Longleftrightarrow P^{(\beta)}(\alpha) \geq 0$.

(ii) $\{\alpha\}$ is big $\Longleftrightarrow P^{(\beta)}(\alpha)>0$.

Proof. ( $i$ ) follows at once from (20) and so does (ii) after we (trivially) notice that the class $\{\alpha\}$ is big iff there exists $\varepsilon>0$ such that $\{\alpha\}-\varepsilon\{\beta\}$ is psef. Indeed, this is a consequence of the fixed class $\{\beta\}$ being supposed big.

Next, we observe some easy but useful properties of the $\beta$-directed trace.

Proposition 4.4 Suppose that $\{\beta\} \in H_{B C}^{1,1}(X, \mathbb{R})$ is a fixed big class.

(i) For all classes $\left\{\alpha_{1}\right\},\left\{\alpha_{2}\right\} \in H_{B C}^{1,1}(X, \mathbb{R})$, we have

$$
P^{(\beta)}\left(\alpha_{1}+\alpha_{2}\right) \geq P^{(\beta)}\left(\alpha_{1}\right)+P^{(\beta)}\left(\alpha_{2}\right) .
$$

In particular, $P^{(\beta)}\left(\alpha_{1}\right) \geq P^{(\beta)}\left(\alpha_{2}\right)$ whenever $\left\{\alpha_{1}\right\} \geq$ psef $\left\{\alpha_{2}\right\}$ (in the sense that $\left\{\alpha_{1}-\alpha_{2}\right\}$ is psef). (ii) For any class $\{\alpha\} \in H_{B C}^{1,1}(X, \mathbb{R})$ and any $t \in \mathbb{R}$, we have

$$
P^{(\beta)}(t \alpha)=t P^{(\beta)}(\alpha) \quad \text { and, if } t>0, \quad P^{(t \beta)}(\alpha)=\frac{1}{t} P^{(\beta)}(\alpha) .
$$

(iii) For every big class $\{\alpha\} \in H_{B C}^{1,1}(X, \mathbb{R})$, we have

$$
P^{(\alpha)}(\alpha)=1
$$


Proof. Let $\left\{\alpha_{1}\right\},\left\{\alpha_{2}\right\} \in H_{B C}^{1,1}(X, \mathbb{R})$. Since $\int_{X}\left(\alpha_{1}+\alpha_{2}\right) \wedge \gamma^{n-1}=\int_{X} \alpha_{1} \wedge \gamma^{n-1}+\int_{X} \alpha_{2} \wedge \gamma^{n-1}$ for every $\left[\gamma^{n-1}\right]_{A} \in H_{A}^{n-1, n-1}(X, \mathbb{R})$, we get

$$
\inf \int_{X}\left(\alpha_{1}+\alpha_{2}\right) \wedge \gamma^{n-1} \geq \inf \int_{X} \alpha_{1} \wedge \gamma^{n-1}+\inf \int_{X} \alpha_{1} \wedge \gamma^{n-1}
$$

where the infima are taken over all $\left[\gamma^{n-1}\right] \in S_{\beta}$. This proves $(i)$.

(ii) follows immediately from $\int_{X} t \alpha \wedge \gamma^{n-1}=t \int_{X} \alpha \wedge \gamma^{n-1}$ and from the fact that $\left[\gamma^{n-1}\right]_{A}$ is $(t \beta)$ normalised if and only if $t\left[\gamma^{n-1}\right]_{A}$ is $\beta$-normalised.

(iii) follows from $\int_{X} \alpha \wedge \gamma^{n-1}=1$ for all $\left[\gamma^{n-1}\right]_{A}$ such that $[\alpha]_{B C} \cdot\left[\gamma^{n-1}\right]_{A}=1$.

The next observation deals with the variation of $P^{(\beta)}$ when $\{\beta\}$ varies. As usual, an inequality $\{\alpha\} \geq_{\text {psef }}\{\beta\}$ between real $(1,1)$-classes will mean that the difference class $\{\alpha-\beta\}$ is psef.

Proposition 4.5 Let $\left\{\beta_{1}\right\},\left\{\beta_{2}\right\} \in H_{B C}^{1,1}(X, \mathbb{R})$ be big classes.

(i) If $\left\{\beta_{1}\right\} \geq_{\text {psef }} C\left\{\beta_{2}\right\}$ for some constant $C>0$, then

$$
P^{\left(\beta_{1}\right)} \leq \frac{1}{C} P^{\left(\beta_{2}\right)} \quad \text { on the psef cone } \mathcal{E}_{X} \subset H_{B C}^{1,1}(X, \mathbb{R}) .
$$

(ii) The following inequality holds:

$$
P^{\left(\beta_{2}\right)}\left(\beta_{1}\right) P^{\left(\beta_{1}\right)} \leq P^{\left(\beta_{2}\right)} \text { on the psef cone } \mathcal{E}_{X} \subset H_{B C}^{1,1}(X, \mathbb{R})
$$

Proof. If $\left\{\beta_{1}-C \beta_{2}\right\}$ is psef, then $\int_{X}\left(\beta_{1}-C \beta_{2}\right) \wedge \gamma^{n-1} \geq 0$, i.e. $\left[\beta_{1}\right]_{B C} \cdot\left[\gamma^{n-1}\right]_{A} \geq C\left[\beta_{2}\right]_{B C} \cdot\left[\gamma^{n-1}\right]_{A}$, for all classes $\left[\gamma^{n-1}\right]_{A} \in \mathcal{G}_{X}$. It follows that, for every psef class $\{\alpha\} \in H_{B C}^{1,1}(X, \mathbb{R})$, we have:

$$
\int_{X} \alpha \wedge \frac{\gamma^{n-1}}{\int_{X} \beta_{1} \wedge \gamma^{n-1}} \leq \frac{1}{C} \int_{X} \alpha \wedge \frac{\gamma^{n-1}}{\int_{X} \beta_{2} \wedge \gamma^{n-1}} \quad \text { for all }\left[\gamma^{n-1}\right]_{A} \in \mathcal{G}_{X}
$$

Taking infima over all $\left[\gamma^{n-1}\right]_{A} \in \mathcal{G}_{X}$, we get (24). On the other hand, it follows from (20) that

$$
\left\{\beta_{1}\right\} \geq_{\text {psef }} P^{\left(\beta_{2}\right)}\left(\beta_{1}\right)\left\{\beta_{2}\right\}
$$

which in turn implies (25) thanks to $(24)$ applied with $C=P^{\left(\beta_{2}\right)}\left(\beta_{1}\right)$.

\subsection{The nef threshold}

We now observe that the discussion of the psef threshold in $\S .4 .1$ can be run analogously in the nef context using the following important result of [DP04, Corollary 0.4].

Theorem 4.6 (Demailly-Paun 2004) Let $X$ be a compact Kähler manifold, dim $\mathbb{C}_{\mathbb{C}}=n$. Then the dual of the nef cone $\overline{\mathcal{K}_{X}} \subset H^{1,1}(X, \mathbb{R})$ under the Serre duality is the closed convex cone $\mathcal{N}_{X} \subset$ $H^{n-1, n-1}(X, \mathbb{R})$ generated by classes of currents of the shape $[Y] \wedge \omega^{n-p-1}$, where $Y$ runs over the irreducible analytic subsets of $X$ of any codimension $p=0,1, \ldots, n-1$ and $\{\omega\}$ runs over the Kähler classes of $X$. 
Let $\{\alpha\},\{\beta\} \in H^{1,1}(X, \mathbb{R})$ be arbitrary classes on a compact Kähler $n$-fold $X$. By Theorem 4.6, for any $s \in \mathbb{R}$, the class $\{\alpha-s \beta\}$ is nef iff

$$
\int_{Y} \alpha \wedge \omega^{n-p-1} \geq s \int_{Y} \beta \wedge \omega^{n-p-1}, \quad p=0,1, \ldots, n-1, \operatorname{codim}_{X} Y=p,\{\omega\} \in \mathcal{K}_{X} .
$$

(As usual, $\mathcal{K}_{X}$ denotes the Kähler cone of $X$.) This immediately implies the following statement.

Proposition 4.7 Let $\{\beta\} \in H_{B C}^{1,1}(X, \mathbb{R})$ be any Kähler class on a compact Kähler $n$-fold $X$. The nef threshold of any $\{\alpha\} \in H_{B C}^{1,1}(X, \mathbb{R})$ in the $\{\beta\}$-direction, defined by the first identity below, also satisfies the second identity:

$$
N^{(\beta)}(\alpha):=\inf \int_{Y} \alpha \wedge \omega^{n-p-1}=\sup \{s \in \mathbb{R} / \text { the class }\{\alpha\}-s\{\beta\} \text { is nef }\},
$$

where the infimum is taken over all $p=0,1, \ldots, n-1$, over all the irreducible analytic subsets $Y \subset X$ such that codim $Y=p$ and over all Kähler classes $\{\omega\}$ normalised such that $\int_{Y} \beta \wedge \omega^{n-p-1}=1$. In particular, the set $\{s \in \mathbb{R} /$ the class $\{\alpha\}-s\{\beta\}$ is nef $\}$ equals the interval $\left(-\infty, N^{(\beta)}(\alpha)\right]$.

Thus, we obtain a function $N^{(\beta)}: H_{B C}^{1,1}(X, \mathbb{R}) \rightarrow \mathbb{R}$. It is clear that

$$
N^{(\beta)}(\alpha) \leq P^{(\beta)}(\alpha) \quad \text { for all }\{\alpha\} \in H_{B C}^{1,1}(X, \mathbb{R})
$$

thanks to the supremum characterisations of the two thresholds and to the well-known implication "nef $\Longrightarrow$ psef".

It is precisely in order to ensure that $\int_{Y} \beta \wedge \omega^{n-p-1}>0$, hence that $\{\omega\}$ can be normalised as stated, for any Kähler class $\{\omega\}$ and any $Y \subset X$ that we assumed $\{\beta\}$ to be Kähler. The two-fold characterisations of the nef and the psef thresholds yield at once the following consequence.

Observation 4.8 Suppose that no analytic subset $Y \subset X$ exists except in codimensions 0 and $n$. Then $N^{(\beta)}(\alpha)=P^{(\beta)}(\alpha)$ for all Kähler classes $\{\alpha\},\{\beta\}$.

Proof. If $Y=X$ is the only analytic subset of $X$ of codimension $p<n$, then $N^{(\beta)}(\alpha)=\inf \int_{X} \alpha \wedge \omega^{n-1}$ where the infimum is taken over all the Kähler classes $\{\omega\}$, i.e. over all the Aeppli-Gauduchon classes $\left[\omega^{n-1}\right]_{A}$ representable by the $(n-1)^{s t}$ power of a Kähler metric, normalised such that $\int_{X} \beta \wedge \omega^{n-1}=$ 1. Since these classes form a subset of all the Aeppli-Gauduchon classes $\left[\gamma^{n-1}\right]_{A}$ normalised by $\int_{X} \beta \wedge \gamma^{n-1}=1$, we get $N^{(\beta)}(\alpha) \geq P^{(\beta)}(\alpha)$. However, the reverse inequality always holds, hence equality holds.

An immediate consequence of Proposition 4.7 is the following analogue of Corollary 4.3 for the nef/Kähler context.

Corollary 4.9 (i) Suppose that $\{\beta\} \in H_{B C}^{1,1}(X, \mathbb{R})$ is a fixed Kähler class. For any class $\{\alpha\} \in$ $H_{B C}^{1,1}(X, \mathbb{R})$, the following equivalences hold:

(i) $\{\alpha\}$ is nef $\Longleftrightarrow N^{(\beta)}(\alpha) \geq 0$. 
(ii) $\{\alpha\}$ is Kähler $\Longleftrightarrow N^{(\beta)}(\alpha)>0$.

In particular, if no analytic subset $Y \subset X$ exists except in codimensions 0 and $n$, then the following (actually known, see [Dem92]) equivalences hold:

(a) $\{\alpha\}$ is nef $\Longleftrightarrow\{\alpha\}$ is psef, (b) $\{\alpha\}$ is Kähler $\Longleftrightarrow\{\alpha\}$ is big.

Proof. ( $i$ ) follows at once from (26) and so does (ii) after we (trivially) notice that the class $\{\alpha\}$ is Kähler iff there exists $\varepsilon>0$ such that $\{\alpha\}-\varepsilon\{\beta\}$ is nef. Indeed, this is a consequence of the fixed class $\{\beta\}$ being supposed Kähler and of the Kähler cone being the interior of the nef cone.

We immediately get analogues of Propositios 4.4 and 4.5 for $N^{(\beta)}(\alpha)$ in place of $P^{(\beta)}(\alpha)$ and for the order relation $\geq_{\text {nef }}$ in place of $\geq_{\text {psef }}$, where $\{\alpha\} \geq_{\text {nef }}\{\beta\}$ means that the class $\{\alpha-\beta\}$ is nef.

\subsection{Relations of the psef/nef threshold to the volume}

We now relate the $\beta$-directed trace of a Kähler class $\{\alpha\}$ to the volume of $\{\alpha-\beta\}$.

Proposition 4.10 (i) For every Kähler classes $\{\alpha\},\{\beta\}$ on a compact Kähler $n$-fold $X$, we have:

$$
\frac{\{\alpha\}^{n}}{n\{\alpha\}^{n-1} \cdot\{\beta\}} \stackrel{(a)}{\leq} P^{(\beta)}(\alpha) \stackrel{(b)}{\leq} \frac{\{\alpha\}^{n}}{\{\alpha\}^{n-1} \cdot\{\beta\}} .
$$

In fact, it suffices to suppose that $\{\beta\}$ is big in the inequality (b). In particular, if $\{\alpha\}^{n}-n\{\alpha\}^{n-1} \cdot\{\beta\}>$ 0 , then $P^{(\beta)}(\alpha)>1$ (hence we find again that $\{\alpha-\beta\}$ is big in this case).

(ii) For every Kähler classes $\{\alpha\},\{\beta\}$ such that $\{\alpha\}^{n}-n\{\alpha\}^{n-1} \cdot\{\beta\}>0$, we have:

$$
\operatorname{Vol}(\{\alpha-\beta\}) \geq\left(1-\frac{1}{P^{(\beta)}(\alpha)}\right)^{n}\{\alpha\}^{n} .
$$

Note that the combination of (29) and part (a) of (28) is the volume lower bound (15).

Proof. ( $i$ ) Inequality (b) is trivial: it suffices to choose $\left[\gamma^{n-1}\right]_{A}=t\left[\alpha^{n-1}\right]_{A}$ for the constant $t>0$ satisfying the $\beta$-normalisation condition $[\beta]_{B C} . t\left[\alpha^{n-1}\right]_{A}=1$, i.e. $t=1 /\{\alpha\}^{n-1} \cdot\{\beta\}$, and to use the definition of $P^{(\beta)}(\alpha)$ as an infimum.

Inequality $(a)$ follows from Corollary 3.2 by taking the infimum over all the Gauduchon metrics $\gamma$ normalised by $\int_{X} \beta \wedge \gamma^{n-1}=1$ in (13).

(ii) We saw in the second proof of the lower estimate (15) that (17) holds for every $t \in[0,1]$ such that $\{\alpha\}-(1 / t)\{\beta\}$ is psef. Now, (20) shows that the infimum of all these $t$ is $1 / P^{(\beta)}(\alpha)$. Thus (17) holds for $t=1 / P^{(\beta)}(\alpha)$, yielding (29).

A similar link between the volume and the nef threshold is given in the next result by considering Monge-Ampère equations on analytic subsets $Y \subset X$.

Proposition 4.11 For every Kähler classes $\{\alpha\},\{\beta\}$ on a compact Kähler $n$-fold $X$, we have:

$$
\inf _{\substack{p=0,1, \ldots, n-1, Y \subset X, \operatorname{codim} Y=p}} \frac{\operatorname{Vol}_{Y}(\alpha)}{(n-p)\{\alpha\}^{n-p-1} \cdot\{\beta\} \cdot\{[Y]\}} \stackrel{(a)}{\leq} N^{(\beta)}(\alpha) \stackrel{(b)}{\leq} \inf _{\substack{p=0,1, \ldots, n-1, Y \subset X, \operatorname{codim} Y=p}} \frac{\operatorname{Vol}_{Y}(\alpha)}{\{\alpha\}^{n-p-1} \cdot\{\beta\} \cdot\{[Y]\}}
$$


where the infima are taken over the analytic subsets $Y \subset X$. We have set $\operatorname{Vol}_{Y}(\alpha):=\int_{Y} \alpha^{n-p}=$ $\int_{X} \alpha^{n-p} \wedge[Y]$ and $\{\alpha\}^{n-p-1} \cdot\{\beta\} .\{[Y]\}:=\int_{Y} \alpha^{n-p-1} \wedge \beta=\int_{X} \alpha^{n-p-1} \wedge \beta \wedge[Y]$ (both quantities depending only on $p$ and the classes $\{\alpha\},\{\beta\},\{[Y]\})$.

Proof. Pick any Kähler metrics $\alpha \in\{\alpha\}$ and $\beta \in\{\beta\}$. Let $Y \subset X$ be any analytic subset of arbitrary codimension $p \in\{0,1, \ldots, n-1\}$ and let $\omega$ be any Kähler metric on $X$ normalised such that $\int_{Y} \beta \wedge \omega^{n-p-1}=1$. We can solve the following Monge-Ampère equation:

$$
\widetilde{\alpha}_{Y}^{n-p}=\operatorname{Vol}_{Y}(\alpha) \beta \wedge \omega^{n-p-1} \quad \text { on } Y
$$

in the sense that there exists a $d$-closed (weakly) positive $(1,1)$-current $\widetilde{\alpha}_{Y}$ on $Y$ (cf. Definition 1.2 in [Dem85]) lying in the restricted class $\{\alpha\}_{\mid Y}$ such that $\widetilde{\alpha}_{Y}$ is $C^{\infty}$ on the regular part $Y_{\text {reg }}$ of $Y$. We defer to the end of the proof the explanation of how this follows from results in the literature. We adopt the standard point of view (see [Dem85, §.1]) according to which $C^{\infty}$ forms on a singular variety $Y$ are defined locally as restrictions to $Y_{\text {reg }}$ of $C^{\infty}$ forms on an open subset of some $\mathbb{C}^{N}$ into which $Y$ locally embeds. In what follows, the exterior powers and products involving $\widetilde{\alpha}_{Y}$ are to be understood on $Y_{\text {reg }}$ even when we write $Y$.

If we define $\operatorname{det}_{\omega} \widetilde{\alpha}_{Y}$ by requiring $\widetilde{\alpha}_{Y}^{n-p}=\left(\operatorname{det}_{\omega} \widetilde{\alpha}_{Y}\right) \omega^{n-p}$ on $Y$, then (31) translates to the identity:

$$
\operatorname{det}_{\omega} \widetilde{\alpha}_{Y}=\frac{\operatorname{Vol}_{Y}(\alpha)}{n-p} \Lambda_{\omega} \beta_{\mid Y} \quad \text { on } Y .
$$

Thus, the argument in the proof of Lemma 3.1 can be rerun on $Y$ as follows:

$$
\begin{aligned}
\left(\int_{Y} \widetilde{\alpha}_{Y}\right. & \left.\wedge \omega^{n-p-1}\right)\left(\int_{Y} \widetilde{\alpha}_{Y}^{n-p-1} \wedge \beta\right)=\frac{1}{(n-p)^{2}}\left(\int_{Y}\left(\Lambda_{\omega} \widetilde{\alpha}_{Y}\right) \omega^{n-p}\right)\left(\int_{Y}\left(\Lambda_{\widetilde{\alpha}_{Y}} \beta_{\mid Y}\right)\left(\operatorname{det}_{\omega} \widetilde{\alpha}_{Y}\right) \omega^{n-p}\right) \\
& \geq \frac{1}{(n-p)^{2}}\left(\int_{Y}\left[\left(\Lambda_{\omega} \widetilde{\alpha}_{Y}\right)\left(\Lambda_{\widetilde{\alpha}_{Y}} \beta_{\mid Y}\right)\right]^{\frac{1}{2}}\left(\operatorname{det}_{\omega} \widetilde{\alpha}_{Y}\right)^{\frac{1}{2}} \omega^{n-p}\right)^{2} \\
& \stackrel{(b)}{\geq} \frac{1}{(n-p)^{2}}\left(\int_{Y}\left(\Lambda_{\omega} \beta_{\mid Y}\right)^{\frac{1}{2}}\left(\frac{\operatorname{Vol}_{Y}(\alpha)}{n-p}\right)^{\frac{1}{2}}\left(\Lambda_{\omega} \beta_{\mid Y}\right)^{\frac{1}{2}} \omega^{n-p}\right)^{2} \\
& =\frac{\operatorname{Vol}_{Y}(\alpha)}{n-p}\left(\frac{1}{(n-p)} \int_{Y}\left(\Lambda_{\omega} \beta_{\mid Y}\right) \omega^{n-p}\right)^{2}=\frac{\operatorname{Vol}_{Y}(\alpha)}{n-p}\left(\int_{Y} \beta \wedge \omega^{n-p-1}\right)^{2} \stackrel{(c)}{=} \frac{\operatorname{Vol}_{Y}(\alpha)}{n-p},
\end{aligned}
$$

where $(a)$ is an application of the Cauchy-Schwarz inequality, $(b)$ follows from the pointwise inequality $\left(\Lambda_{\omega} \widetilde{\alpha}_{Y}\right)\left(\Lambda_{\widetilde{\alpha}_{Y}} \beta\right) \geq \Lambda_{\omega} \beta$ (cf. [Pop14, Lemma 3.1]) and from (32), while (c) follows from the normalisation $\int_{X} \beta \wedge \omega^{n-p-1}=1$.

Thus, since $\int_{Y} \widetilde{\alpha}_{Y} \wedge \omega^{n-p-1}=\int_{Y} \alpha \wedge \omega^{n-p-1}$ and $\int_{Y} \widetilde{\alpha}_{Y}^{n-p-1} \wedge \beta=\int_{Y} \alpha^{n-p-1} \wedge \beta$, we get:

$$
\int_{Y} \alpha \wedge \omega^{n-p-1} \geq \frac{\operatorname{Vol}_{Y}(\alpha)}{(n-p)\{\alpha\}^{n-p-1} \cdot\{\beta\} \cdot\{[Y]\}}
$$

for every analytic subset $Y \subset X$ and every Kähler metric $\omega$ normalised by $\int_{Y} \beta \wedge \omega^{n-p-1}=1$. This proves inequality $(a)$ in $(30)$. 
The proof of inequality $(b)$ in (30) follows immediately by choosing the Kähler metric $\omega$ to be proportional to $\alpha$, i.e. $\omega=t \alpha$ for the constant $t=t_{Y}>0$ determined by the normalisation condition $\int_{Y} \beta \wedge \omega^{n-p-1}=1$ once $Y \subset X$ has been chosen. Indeed, for every $p=0,1, \ldots, n-1$ and every analytic subset $Y \subset X$, we immediately get:

$$
\inf _{\omega} \int_{Y} \alpha \wedge \omega^{n-p-1} \leq \int_{Y} \alpha \wedge(t \alpha)^{n-p-1}=\frac{\int_{Y} \alpha^{n-p}}{\int_{Y} \beta \wedge \alpha^{n-p-1}}
$$

which implies part (b) of (30) after taking the infimum over $p$ and $Y$.

It remains to explain how the solution of equation (31) is obtained. If $Y$ is smooth, Yau's classical theorem in [Yau78] ensures the existence and uniqueness of a Kähler metric $\widetilde{\alpha}_{Y}$ in $\{\alpha\}_{\mid Y}$ which solves (31). If $Y$ is singular, we choose a desingularisation $\tilde{Y}$ of $Y$ that is a finite sequence of blow-ups with smooth centres in $X$ :

$$
\mu: \tilde{Y} \longrightarrow Y, \text { which is the restriction of } \mu: \tilde{X} \longrightarrow X .
$$

Thus, $\mu: \tilde{X} \backslash \mu^{-1}(Z) \longrightarrow X \backslash Z$ is a biholomorphism above the complement of the analytic subset $Z:=Y_{\text {sing }}$ and $\widetilde{X}$ is a compact Kähler manifold, hence so is the submanifold $\widetilde{Y}$. Moreover, $\mu^{\star}\left(\beta \wedge \omega^{n-p-1}\right)$ is a $C^{\infty}$ semi-positive $(n-p, n-p)$-form on $\tilde{X}$ that is strictly positive on $\tilde{X} \backslash \mu^{-1}(Z)$. Clearly, $\mu^{\star}\{\alpha\}=\left\{\mu^{\star} \alpha\right\}$ is a semi-positive (hence also nef) big class on $\widetilde{X}$ and

$$
\operatorname{Vol}_{\widetilde{Y}}\left(\mu^{\star}\{\alpha\}\right)=\int_{\widetilde{X}}\left(\mu^{\star} \alpha\right)^{n-p} \wedge[\widetilde{Y}]=\int_{X} \alpha^{n-p} \wedge[Y]=\operatorname{Vol}_{Y}(\alpha)>0 .
$$

We consider the following Monge-Ampère equation on the (smooth) compact Kähler manifold $\widetilde{Y}$ :

$$
\widetilde{\alpha}_{\widetilde{Y}}^{n-p}=\operatorname{Vol}_{\widetilde{Y}}\left(\mu^{\star}\{\alpha\}\right) \mu^{\star}\left(\beta \wedge \omega^{n-p-1}\right) \quad \text { on } \tilde{Y}
$$

If the class $\mu^{\star}\{\alpha\}$ were Kähler, Yau's Theorem 3 in [Yau78] on solutions of the Monge-Amère equation with a degenerate (i.e. semi-positive) smooth r.h.s. would yield a unique $d$-closed $(1,1)$ current $\widetilde{\alpha}_{\widetilde{Y}} \in \mu^{\star}\{\alpha\}_{\mid \widetilde{Y}}$ solving equation (33) such that $\widetilde{\alpha}_{\widetilde{Y}} \geq 0$ on $\widetilde{Y}, \widetilde{\alpha}_{\widetilde{Y}}$ is $C^{\infty}$ on $\widetilde{Y} \backslash \mu^{-1}(Z)$ and $\widetilde{\alpha}_{\widetilde{Y}}$ has locally bounded coefficients on $\widetilde{Y}$. In our more general case where the class $\mu^{\star}\{\alpha\}$ is only semi-positive and big, Theorems A, B, C in [BEGZ10] yield a unique $d$-closed $(1,1)$-current $\widetilde{\alpha}_{\widetilde{Y}} \in \mu^{\star}\{\alpha\}_{\mid \widetilde{Y}}$ such that $\widetilde{\alpha}_{\widetilde{Y}} \geq 0$ on $\widetilde{Y}$ and

$$
\left\langle\widetilde{\alpha}_{\widetilde{Y}}^{n-p}\right\rangle=\operatorname{Vol}_{\widetilde{Y}}\left(\mu^{\star}\{\alpha\}\right) \mu^{\star}\left(\beta \wedge \omega^{n-p-1}\right) \quad \text { on } \tilde{Y}
$$

where \langle\rangle stands for the non-pluripolar product introduced in [BEGZ10]. Moreover, $\widetilde{\alpha}_{\widetilde{Y}}$ is $C^{\infty}$ on the ample locus of the class $\mu^{\star}\{\alpha\}_{\mid \widetilde{Y}}$ (cf. Theorem $\mathrm{C}$ in [BEGZ10]), which in our case coincides with $\widetilde{Y} \backslash \mu^{-1}(Z)$, and $\widetilde{\alpha}_{\tilde{Y}}$ has minimal singularities (cf. Theorem B in [BEGZ10]) among the positive currents in the class $\mu^{\star}\{\alpha\}_{\mid \widetilde{Y}}$. Since this class contains $C^{\infty}$ semi-positive forms $\left(\right.$ e.g. $\left.\left(\mu^{\star} \alpha\right)_{\mid \widetilde{Y}}\right)$, its currents with minimal singularties have locally bounded potentials. Thus, $\widetilde{\alpha}_{\widetilde{Y}}$ has locally bounded (and even continuous) potentials, so $\left\langle\widetilde{\alpha}_{\widetilde{Y}}^{n-p}\right\rangle$ equals the exterior power $\widetilde{\alpha}_{\widetilde{Y}}^{n-p}$ in the sense of Bedford and 
Taylor [BT82]. In particular, $\left[\widetilde{\alpha}_{\widetilde{Y}}^{k}\right]_{B C}=\left[\mu^{\star} \alpha^{k}\right]_{B C}$ for all $k$, so $\int_{\widetilde{Y}} \widetilde{\alpha}_{\widetilde{Y}}^{n-p-1} \wedge \mu^{\star} \beta=\int_{\widetilde{Y}} \mu^{\star} \alpha^{n-p-1} \wedge \mu^{\star} \beta$. It remains to set

$$
\widetilde{\alpha}_{Y}:=\mu_{\star} \widetilde{\alpha}_{\widetilde{Y}}
$$

We thus get a $d$-closed positive $(1,1)$-current $\widetilde{\alpha}_{Y} \in\{\alpha\}_{\mid Y}$ whose restriction to $Y_{\text {reg }}=Y \backslash Z$ is $C^{\infty}$ and which solves the Monge-Ampère equation (31).

We can now relate both the psef and the nef thresholds $P^{(\beta)}(\alpha), N^{(\beta)}(\alpha)$ to the volume of $\{\alpha-\beta\}$. The next result confirms Conjecture 1.1 in the case when these thresholds are sufficiently close to each other.

Proposition 4.12 Let $X$ be a compact Kähler manifold, $\operatorname{dim}_{\mathbb{C}} X=n$, and let $\{\alpha\},\{\beta\} \in H_{B C}^{1,1}(X, \mathbb{R})$ be Kähler classes such that

$$
\{\alpha\}^{n}-n\{\alpha\}^{n-1} \cdot\{\beta\}>0 .
$$

If either of the following two conditions is satisfied:

$$
\text { (i) } N^{(\beta)}(\alpha) \geq 1 \quad \text { or } \quad \text { (ii) } N^{(\beta)}(\alpha) \geq \frac{\frac{\{\alpha\}^{n}}{\{\alpha\}^{n-1} \cdot\{\beta\}}-P^{(\beta)}(\alpha)}{n-1},
$$

then

$$
\operatorname{Vol}(\{\alpha-\beta\}) \geq\{\alpha\}^{n}-n\{\alpha\}^{n-1} \cdot\{\beta\} .
$$

Note that $P^{(\beta)}(\alpha) \geq \frac{\frac{\{\alpha\}^{n}}{\{\alpha\}^{n-1} \cdot\{\beta\}}-P^{(\beta)}(\alpha)}{n-1}$ thanks to inequality $(a)$ in $(28)$. Since $P^{(\beta)}(\alpha) \geq N^{(\beta)}(\alpha)$ (cf. (27)), this shows that condition $(i i)$ requires $N^{(\beta)}(\alpha)$ to be "close" to $P^{(\beta)}(\alpha)$. In particular, (ii) holds if $N^{(\beta)}(\alpha)$ and $P^{(\beta)}(\alpha)$ coincide.

Proof of Proposition 4.12. If $N^{(\beta)}(\alpha) \geq 1$, then the class $\{\alpha-\beta\}$ is nef (cf. Proposition 4.7), so (36) follows from Proposition 2.2 in this case.

Let us now suppose that $N^{(\beta)}(\alpha)<1$ and that condition $(i i)$ is satisfied. We set $s_{0}:=N^{(\beta)}(\alpha)$ and $t_{0}:=P^{(\beta)}(\alpha)$, so $s_{0}<1<t_{0}$ (where the last inequality follows from $\{\alpha-\beta\}$ being big - the main result in $[\mathrm{Pop} 14])$. We have

$$
\{\alpha-\beta\}=\frac{t_{0}-1}{t_{0}-s_{0}}\left\{\alpha-s_{0} \beta\right\}+\frac{1-s_{0}}{t_{0}-s_{0}}\left\{\alpha-t_{0} \beta\right\} .
$$

Since the class $\left(1-s_{0}\right) /\left(t_{0}-s_{0}\right) \cdot\left\{\alpha-t_{0} \beta\right\}$ is psef, we get the first inequality below:

$$
\operatorname{Vol}(\{\alpha-\beta\}) \geq\left(\frac{t_{0}-1}{t_{0}-s_{0}}\right)^{n} \operatorname{Vol}\left(\left\{\alpha-s_{0} \beta\right\}\right) \geq\left(1-\frac{1-s_{0}}{t_{0}-s_{0}}\right)^{n}\left(\{\alpha\}^{n}-n s_{0}\{\alpha\}^{n-1} \cdot\{\beta\}\right)
$$

where the second inequality follows from Proposition 2.2 since the class $\left\{\alpha-s_{0} \beta\right\}$ is nef. Let 


$$
f:[0,1] \rightarrow[0,+\infty), \quad f(s):=\left(1-\frac{1-s}{t_{0}-s}\right)^{n}\left(\{\alpha\}^{n}-n s\{\alpha\}^{n-1} \cdot\{\beta\}\right) .
$$

Thus $f(1)=\{\alpha\}^{n}-n\{\alpha\}^{n-1} \cdot\{\beta\}$ and (38) translates to $\operatorname{Vol}(\{\alpha-\beta\}) \geq f\left(s_{0}\right)$.

We will now show that $f$ is non-increasing on the interval $\left[\frac{R-t_{0}}{n-1}, 1\right]$, where we set:

$$
R:=\frac{\{\alpha\}^{n}}{\{\alpha\}^{n-1} \cdot\{\beta\}} \quad \text { or equivalently } \quad R=\sup \left\{r>0 /\{\alpha\}^{n}-r\{\alpha\}^{n-1} \cdot\{\beta\}>0\right\} .
$$

Assumption (34) means that $R>n$. Deriving $f$, we get:

$$
\begin{aligned}
f^{\prime}(s) & =-n \frac{\left(t_{0}-1\right)^{n}}{\left(t_{0}-s\right)^{n}}\{\alpha\}^{n-1} \cdot\{\beta\}+n \frac{\left(t_{0}-1\right)^{n-1}}{\left(t_{0}-s\right)^{n-1}} \frac{t_{0}-1}{\left(t_{0}-s\right)^{2}}\left(\{\alpha\}^{n}-n s\{\alpha\}^{n-1} \cdot\{\beta\}\right) \\
& =n \frac{\left(t_{0}-1\right)^{n}}{\left(t_{0}-s\right)^{n+1}}\left(\{\alpha\}^{n}-\left((n-1) s+t_{0}\right)\{\alpha\}^{n-1} \cdot\{\beta\}\right), \quad s \in[0,1] .
\end{aligned}
$$

Since $t_{0}-1>0$ and $t_{0}-s>0$, the definition of $R$ implies that $f^{\prime}(s) \leq 0$ for all $s$ such that $(n-1) s+t_{0} \geq R$, i.e. for all $s \geq \frac{R-t_{0}}{n-1}$.

Recall that we are working under the assumption $s_{0} \in\left[\frac{R-t_{0}}{n-1}, 1\right)$, so from $f$ being non-increasing on $\left[\frac{R-t_{0}}{n-1}, 1\right]$ we infer that $f\left(s_{0}\right) \geq f(1)=\{\alpha\}^{n}-n\{\alpha\}^{n-1} \cdot\{\beta\}$. Since $\operatorname{Vol}(\{\alpha-\beta\}) \geq f\left(s_{0}\right)$ by (38), we get $(36)$.

\subsection{Nef/psef thresholds and volume revisited}

We now prove Theorem 1.4. In so doing, we use a different method for obtaining a lower bound for the volume of $\{\alpha-\beta\}$ that takes into account the "angles" between $\left\{\alpha-s_{0} \beta\right\}$ and $\{\alpha-t \beta\}$ when $t$ varies in a subinterval of $\left[1, t_{0}\right)$.

We start with a useful observation in linear algebra generalising inequality (6).

Lemma 4.13 Let $\alpha>0$ and $\beta \geq 0$ be $C^{\infty}(1,1)$-forms on an arbitrary complex manifold $X$ with $\operatorname{dim}_{\mathbb{C}} X=n$ such that $\alpha-\beta \geq 0$. Then, for every $k \in\{0,1, \ldots, n\}$, the following inequality holds:

$$
(\alpha-\beta)^{n-k} \wedge \alpha^{k} \geq \alpha^{n}-(n-k) \alpha^{n-1} \wedge \beta
$$

Proof. Let $x_{0} \in X$ be any point and $z_{1}, \ldots, z_{n}$ local holomorphic coordinates about $x_{0}$ such that

$$
\alpha=\sum_{j=1}^{n} i d z_{j} \wedge d \bar{z}_{j} \quad \text { and } \quad \beta=\sum_{j=1}^{n} \beta_{j} i d z_{j} \wedge d \bar{z}_{j}, \quad \text { hence } \quad \alpha-\beta=\sum_{j=1}^{n}\left(1-\beta_{j}\right) i d z_{j} \wedge d \bar{z}_{j} \quad \text { at } x_{0} .
$$

Thus $\beta_{j} \in[0,1]$ for all $j=1, \ldots, n$ by our assumptions and inequality $(41)$ at $x_{0}$ translates to

$$
\frac{k !(n-k) !}{n !} \sum_{1 \leq j_{1}<\cdots<j_{n-k} \leq n}\left(1-\beta_{j_{1}}\right) \ldots\left(1-\beta_{j_{n-k}}\right) \geq 1-\frac{n-k}{n} \sum_{l=1}^{n} \beta_{l},
$$


which, in turn, translates to the following inequality after we set $\gamma_{j}:=1-\beta_{j} \in[0,1]$ :

$$
\frac{k !(n-k) !}{n !}\left(\sum_{1 \leq j_{1}<\cdots<j_{k} \leq n} \frac{1}{\gamma_{j_{1}} \ldots \gamma_{j_{k}}}\right) \gamma_{1} \ldots \gamma_{n} \geq \frac{n-k}{n} \sum_{l=1}^{n} \gamma_{l}+k+1-n .
$$

Note that the l.h.s. of (42) is meaningful even if some $\gamma_{j}$ vanishes because it reappears in $\gamma_{1} \ldots \gamma_{n}$. We will prove inequality (42) by induction on $n \geq 1$ (where $k \in\{1, \ldots, n\}$ is fixed arbitrarily).

If $n=1$, (42) reads $1 \geq 1$. Although it is not required by the induction procedure, we now prove (42) for $n=3$ and $k=1$ since this case will be used further down, i.e. we prove

$$
\frac{1}{3}\left(\gamma_{1} \gamma_{2}+\gamma_{2} \gamma_{3}+\gamma_{3} \gamma_{1}\right) \geq \frac{2}{3}\left(\gamma_{1}+\gamma_{2}+\gamma_{3}\right)-1 \quad \text { for all } \gamma_{1}, \gamma_{2}, \gamma_{3} \in[0,1]
$$

It is clear that (43) is equivalent to $\left(\gamma_{1}-1\right)\left(\gamma_{2}-1\right)+\left(\gamma_{2}-1\right)\left(\gamma_{3}-1\right)+\left(\gamma_{3}-1\right)\left(\gamma_{1}-1\right) \geq 0$ which clearly holds since $\gamma_{j}-1 \leq 0$ for all $j$.

Now we perform the induction step. Suppose that we have proved (42) for all $1 \leq m \leq n$. Proving (42) for $n+1$ amounts to proving the following inequality:

$$
A_{k, n+1}:=\frac{k !(n+1-k) !}{(n+1) !} \sum_{1 \leq j_{1}<\cdots<j_{k} \leq n+1} \frac{\gamma_{1} \cdots \gamma_{n+1}}{\gamma_{j_{1}} \cdots \gamma_{j_{k}}} \geq \frac{n+1-k}{n+1} \sum_{l=1}^{n+1} \gamma_{l}+k-n .
$$

The left-hand term $A_{k, n+1}$ of (44) can be re-written as

$\frac{k !(n+1-k) !}{(n+1) !} \frac{1}{n+1-k}\left(\gamma_{1} \sum_{\substack{\neq 11 \\ 1 \leq r_{1}<\cdots<r_{n-k} \leq n+1}} \gamma_{r_{1}} \cdots \gamma_{r_{n-k}}+\cdots+\gamma_{n+1} \sum_{\substack{\neq=n+1 \\ 1 \leq r_{1}<\cdots<r_{n-k} \leq n+1}} \gamma_{r_{1}} \cdots \gamma_{r_{n-k}}\right)$

where the meaning of the notation is that the sum whose coefficient is $\gamma_{s}$ runs over all the ordered indices $r_{1}<\cdots<r_{n-k}$ selected from the set $\{1, \ldots, n+1\} \backslash\{s\}$. Now, using inequality (42) for $n$ (the induction hypothesis), for every $s \in\{1, \ldots, n+1\}$ we get:

$$
\sum_{\substack{\neq s \\ 1 \leq r_{1}<\cdots<r_{n-k} \leq n+1}} \gamma_{r_{1}} \ldots \gamma_{r_{n-k}} \geq \frac{n !}{k !(n-k) !}\left(\frac{n-k}{n} \sum_{l \in\{1, \ldots, n+1\} \backslash\{s\}} \gamma_{l}+k+1-n\right) .
$$

Plugging these inequalities into the last (re-written) expression for $A_{k, n+1}$, we get:

$$
A_{k, n+1} \geq \frac{n-k}{n(n+1)}\left(\sum_{l \in\{1, \ldots, n+1\} \backslash\{1\}} \gamma_{1} \gamma_{l}+\cdots+\sum_{l \in\{1, \ldots, n+1\} \backslash\{n+1\}} \gamma_{n+1} \gamma_{l}\right)+\frac{k+1-n}{n+1}\left(\gamma_{1}+\cdots+\gamma_{n+1}\right)
$$


hence

$$
\begin{aligned}
A_{k, n+1} & \geq \frac{2(n-k)}{n(n+1)} \sum_{1 \leq j<k \leq n+1} \gamma_{j} \gamma_{k}+\frac{k+1-n}{n+1} \sum_{l=1}^{n+1} \gamma_{l} \\
& =\frac{2(n-k)}{n(n+1)} \frac{\sum_{1 \leq j<k<l \leq n+1}\left(\gamma_{j} \gamma_{k}+\gamma_{k} \gamma_{l}+\gamma_{l} \gamma_{j}\right)}{n-1}+\frac{k+1-n}{n+1} \sum_{l=1}^{n+1} \gamma_{l} \\
& \stackrel{(a)}{\geq} \frac{2(n-k)}{(n-1) n(n+1)}\left(2 \sum_{1 \leq j<k<l \leq n+1}\left(\gamma_{j}+\gamma_{k}+\gamma_{l}\right)-3\left(\begin{array}{c}
n+1 \\
3
\end{array}\right)\right)+\frac{k+1-n}{n+1} \sum_{l=1}^{n+1} \gamma_{l} \\
& =\frac{2(n-k)}{(n-1) n(n+1)}\left(2\left(\begin{array}{c}
n \\
2
\end{array}\right) \sum_{l=1}^{n+1} \gamma_{l}-3\left(\begin{array}{c}
n+1 \\
3
\end{array}\right)\right)+\frac{k+1-n}{n+1} \sum_{l=1}^{n+1} \gamma_{l} \\
& =\frac{1}{n+1}\left(\frac{4(n-k)}{n(n-1)} \frac{n(n-1)}{2}+k+1-n\right) \sum_{l=1}^{n+1} \gamma_{l}-\frac{2(n-k)}{(n-1) n(n+1)} 3 \frac{(n-1) n(n+1)}{2 \cdot 3} \\
& =\frac{n-k+1}{n+1} \sum_{l=1}^{n+1} \gamma_{l}-(n-k),
\end{aligned}
$$

where inequality (a) above follows from (43) applied to each sum $\gamma_{j} \gamma_{k}+\gamma_{k} \gamma_{l}+\gamma_{l} \gamma_{j}$. Thus we have got precisely the inequality (44) that we set out to prove. The proof of Lemma 4.13 is complete.

Now suppose we are in the setting of Conjecture 1.1. We keep the notation of $\S .4 .3$. Recall that $s_{0}:=N^{(\beta)}(\alpha)$ and $t_{0}:=P^{(\beta)}(\alpha)$. We assume that $s_{0}<1$ (since Conjecture 1.1 has been proved in the case when $s_{0} \geq 1$ ).

We express the class $\{\alpha-\beta\}$ as a convex combination of the nef class $\left\{\alpha-s_{0} \beta\right\}$ and the big class $\{\alpha-t \beta\}$ for every $t \in\left[1, t_{0}\right)$ (cf. Theorem 3.5) in the following more flexible version of (37):

$$
\{\alpha-\beta\}=\frac{t-1}{t-s_{0}}\left\{\alpha-s_{0} \beta\right\}+\frac{1-s_{0}}{t-s_{0}}\{\alpha-t \beta\}, \quad t \in\left[1, t_{0}\right) .
$$

We know from Theorem 3.5 that for every $t<\frac{R}{n}$ (cf. notation (40)) there exists a Kähler current $T_{t}$ in the class $\{\alpha-t \beta\}$ such that $T_{t} \geq\left(1-\frac{n}{R} t\right) \alpha$. Thus we get the following Kähler current in the class $\{\alpha-\beta\}$ :

$$
S_{t}:=\frac{t-1}{t-s_{0}}\left(\alpha-s_{0} \beta\right)+\frac{1-s_{0}}{t-s_{0}} T_{t} \geq \frac{t-1}{t-s_{0}}\left(\alpha-s_{0} \beta\right)+\frac{1-s_{0}}{t-s_{0}}\left(1-\frac{n}{R} t\right) \alpha, \quad t \in\left[1, \frac{R}{n}\right],
$$

since the class $\left\{\alpha-s_{0} \beta\right\}$ being nef allows us to assume without loss of generality that $\alpha-s_{0} \beta \geq 0$ (after possibly adding $\varepsilon \omega$ and letting $\varepsilon \downarrow 0$ in the end). Since the r.h.s. of (46) is smooth, it also provides a lower bound for the absolutely continuous part of $S_{t}$, so we get the following lower bound for the volume for all $t \in\left[1, \frac{R}{n}\right]$ :

$$
\operatorname{Vol}(\{\alpha-\beta\}) \geq \int_{X} S_{t, a c}^{n} \geq \frac{1}{\left(t-s_{0}\right)^{n}} \sum_{k=0}^{n}\left(\begin{array}{l}
n \\
k
\end{array}\right)(t-1)^{n-k}\left(1-s_{0}\right)^{k}\left(1-\frac{n t}{R}\right)^{k} \int_{X}\left(\alpha-s_{0} \beta\right)^{n-k} \wedge \alpha^{k}
$$


Since the class $\left\{\alpha-s_{0} \beta\right\}$ is nef, using Lemma 4.13, we get the following

Lemma 4.14 Let $X$ be a compact Kähler manifold, $\operatorname{dim}_{\mathbb{C}} X=n$, and let $\{\alpha\},\{\beta\} \in H_{B C}^{1,1}(X, \mathbb{R})$ be Kähler classes such that $\{\alpha\}^{n}-n\{\alpha\}^{n-1} \cdot\{\beta\}>0$. Suppose that $s_{0}:=N^{(\beta)}(\alpha)<1$. Then the following estimate holds:

$$
\operatorname{Vol}(\{\alpha-\beta\}) \geq\left(\frac{A t-s_{0}}{t-s_{0}}\right)^{n}\left(\{\alpha\}^{n}-\frac{s_{0}(t-1)}{A t-s_{0}} n\{\alpha\}^{n-1} \cdot\{\beta\}\right), \text { for all } t \in\left[1, \frac{R}{n}\right],
$$

where we denote $R:=\{\alpha\}^{n} /\{\alpha\}^{n-1} .\{\beta\}>n$ and $A:=1-\frac{n}{R}\left(1-s_{0}\right) \in\left(s_{0}, 1\right)$.

Proof. From $A-s_{0}=\left(1-s_{0}\right)\left(1-\frac{n}{R}\right) \in(0,1)$ (because $s_{0} \in(0,1)$ and $\left.1-\frac{n}{R} \in(0,1)\right)$, we infer that $A>s_{0}$. That $A<1$ is obvious.

Without loss of generality, we may assume that $\alpha-s_{0} \beta \geq 0$, so (41) applies to $\alpha$ and $s_{0} \beta$ and from (47) we get:

$$
\begin{aligned}
\mathrm{Vol}(\{\alpha-\beta\}) \geq & \frac{1}{\left(t-s_{0}\right)^{n}} \sum_{k=0}^{n}\left(\begin{array}{l}
n \\
k
\end{array}\right)(t-1)^{n-k}\left(1-s_{0}\right)^{k}\left(1-\frac{n t}{R}\right)^{k}\left(\{\alpha\}^{n}-(n-k) s_{0}\{\alpha\}^{n-1} \cdot\{\beta\}\right) \\
= & \frac{1}{\left(t-s_{0}\right)^{n}}\left[t-1+\left(1-s_{0}\right)\left(1-\frac{n t}{R}\right)\right]^{n}\{\alpha\}^{n} \\
& \quad-\frac{t-1}{\left(t-s_{0}\right)^{n}}\left[t-1+\left(1-s_{0}\right)\left(1-\frac{n t}{R}\right)\right]^{n-1} n s_{0}\{\alpha\}^{n-1} \cdot\{\beta\},
\end{aligned}
$$

which proves $(48)$ since $t-1+\left(1-s_{0}\right)\left(1-\frac{n t}{R}\right)=A t-s_{0}$.

Thus, it becomes necessary to study the variation of the folowing function:

$$
g:\left[1, \frac{R}{n}\right] \rightarrow \mathbb{R}, \quad g(t):=\left(\frac{A t-s_{0}}{t-s_{0}}\right)^{n}\left(\{\alpha\}^{n}-\frac{s_{0}(t-1)}{A t-s_{0}} n\{\alpha\}^{n-1} \cdot\{\beta\}\right),
$$

since $\operatorname{Vol}(\{\alpha-\beta\}) \geq g(t)$ for all $t \in\left[1, \frac{R}{n}\right]$. From $A-s_{0}=\left(1-s_{0}\right)\left(1-\frac{n}{R}\right) \in(0,1)$, we get:

$$
g(1)=\left(1-\frac{n}{R}\right)^{n}\{\alpha\}^{n}, \text { while } g\left(\frac{R}{n}\right)=\left(\frac{R-n}{R-n s_{0}}\right)^{n}\left(\{\alpha\}^{n}-n s_{0}\{\alpha\}^{n-1} \cdot\{\beta\}\right) .
$$

We see that $g(1)$ is precisely the lower bound obtained for the volume of $\{\alpha-\beta\}$ in (15), so this lower bound will be improved if $g(t)>g(1)$ for some $t \in(1, R / n]$.

Variation of $g$. Since $\left[(t-1) /\left(A t-s_{0}\right)\right]^{\prime}=\left(A-s_{0}\right) /\left(A t-s_{0}\right)^{2}$ and $\left[\left(A t-s_{0}\right) /\left(t-s_{0}\right)\right]^{\prime}=$ $(1-A) s_{0} /\left(t-s_{0}\right)^{2}$, for the derivative of $g(t)$ we get: $g^{\prime}(t)=$

$$
n(1-A) s_{0} \frac{\left(A t-s_{0}\right)^{n-1}}{\left(t-s_{0}\right)^{n+1}}\left(\{\alpha\}^{n}-\frac{\left(n s_{0}-n s_{0} A+A-s_{0}\right) t-n s_{0}(1-A)-s_{0}\left(A-s_{0}\right)}{(1-A)\left(A t-s_{0}\right)}\{\alpha\}^{n-1} \cdot\{\beta\}\right) .
$$


Now, $A t-s_{0}>0$ for all $t \in[1, R / n]$ since $A t-s_{0} \geq A-s_{0}=\left(1-s_{0}\right)\left(1-\frac{n}{R}\right)>0$. Since $t \geq 1>s_{0}$, from the definition (40) of $R$, we get the equivalences:

$$
\begin{aligned}
g^{\prime}(t) \geq 0 & \Longleftrightarrow\left[n s_{0}(1-A)+A-s_{0}\right] t-n s_{0}(1-A)-s_{0}\left(A-s_{0}\right) \leq(1-A)\left(A t-s_{0}\right) R(51) \\
& \Longleftrightarrow-\left[R A^{2}-\left(n s_{0}-1+R\right) A+(n-1) s_{0}\right] t+s_{0}\left[A-s_{0}+(n-R)(1-A)\right] \geq 0
\end{aligned}
$$

- Sign of $R A^{2}-\left(n s_{0}-1+R\right) A+(n-1) s_{0}$. The discriminant of this $2^{\text {nd }}$ degree polynomial in $A$ is

$$
\Delta_{R}=R^{2}-2\left((n-2) s_{0}+1\right) R+\left(n s_{0}-1\right)^{2} .
$$

The discriminant of $\Delta_{R}$ (viewed as a polynomial in $R$ ) is

$$
\Delta^{\prime}=16(n-1) s_{0}\left(1-s_{0}\right)>0 \quad \text { since } s_{0} \in(0,1)
$$

Thus, the $\Delta_{R}$ vanishes at $R_{1}=(n-2) s_{0}+1-2 \sqrt{(n-1) s_{0}\left(1-s_{0}\right)}$ and $R_{2}=(n-2) s_{0}+1+$ $2 \sqrt{(n-1) s_{0}\left(1-s_{0}\right)}$.

Lemma 4.15 With our usual notation $R:=\{\alpha\}^{n} /\{\alpha\}^{n-1} \cdot\{\beta\}$, we have: $R_{1}<R_{2} \leq n<R$.

Proof. Only the inequality $R_{2} \leq n$ needs a proof. It is equivalent to

$$
\begin{aligned}
(n-2) s_{0}+2 \sqrt{(n-1) s_{0}\left(1-s_{0}\right)} \leq n-1 & \Longleftrightarrow 2 \sqrt{(n-1) s_{0}\left(1-s_{0}\right)} \leq(n-1)\left(1-s_{0}\right)+s_{0} \\
& \Longleftrightarrow\left(\sqrt{(n-1)\left(1-s_{0}\right)}-\sqrt{s_{0}}\right)^{2} \geq 0,
\end{aligned}
$$

which clearly holds.

The upshot is that $\Delta_{R}>0$, so $R A^{2}-\left(n s_{0}-1+R\right) A+(n-1) s_{0}$ vanishes at $A_{1}=\left(n s_{0}-1+\right.$ $\left.R-\sqrt{\Delta_{R}}\right) / 2 R$ and $A_{2}=\left(n s_{0}-1+R+\sqrt{\Delta_{R}}\right) / 2 R$.

Lemma 4.16 With our notation $A:=1-\frac{n}{R}\left(1-s_{0}\right) \in[0,1)$, we have: $A_{1}<A<A_{2}$.

Proof. The inequality $A_{1}<A$ is equivalent to

$$
\frac{n s_{0}-1+R-\sqrt{\Delta_{R}}}{2 R}<\frac{R-n+n s_{0}}{R} \Longleftrightarrow n\left(2-s_{0}\right)-1-R<\sqrt{\Delta_{R}} .
$$

If $n\left(2-s_{0}\right)-1-R \leq 0,(54)$ is obvious. If $n\left(2-s_{0}\right)-1-R>0$, inequality (54) is equivalent to

$$
\begin{aligned}
R^{2}+\left[n\left(2-s_{0}\right)-1\right]^{2}-2\left[n\left(2-s_{0}\right)-1\right] R & <R^{2}-2\left[(n-2) s_{0}+1\right] R+\left(n s_{0}-1\right)^{2} \Longleftrightarrow \\
{\left[n\left(2-s_{0}\right)-n s_{0}\right]\left[n\left(2-s_{0}\right)+n s_{0}+2\right] } & <2\left[n\left(2-s_{0}\right)-(n-2) s_{0}-2\right] R \\
n(n-1)\left(1-s_{0}\right)<\left(1-s_{0}\right)(n-1) R \Longleftrightarrow n & <R,
\end{aligned}
$$

where the last inequality holds thanks to our assumption $\{\alpha\}^{n}-n\{\alpha\}^{n-1} .\{\beta\}>0$.

The inequality $A<A_{2}$ is equivalent to 


$$
\frac{R-n+n s_{0}}{R}<\frac{n s_{0}-1+R+\sqrt{\Delta_{R}}}{2 R} \Longleftrightarrow R+1-\left(2-s_{0}\right) n<\sqrt{\Delta_{R}} .
$$

If $R+1-\left(2-s_{0}\right) n \leq 0$, (55) is obvious. If $R+1-\left(2-s_{0}\right) n>0$, inequality (55) is equivalent to

$$
\begin{aligned}
R^{2}+\left[1-\left(2-s_{0}\right) n\right]^{2}+2\left[1-\left(2-s_{0}\right) n\right] R & <R^{2}-2\left[(n-2) s_{0}+1\right] R+\left(n s_{0}-1\right)^{2} \Longleftrightarrow \\
2\left[2-\left(2-s_{0}\right) n+(n-2) s_{0}\right] R & <\left[n s_{0}-\left(2-s_{0}\right) n\right]\left[n s_{0}-2+\left(2-s_{0}\right) n\right] \Longleftrightarrow \\
(n-1)\left(s_{0}-1\right) R<n(n-1)\left(s_{0}-1\right) \Longleftrightarrow R & >n \quad \text { since } s_{0}-1<0,
\end{aligned}
$$

where the last inequality holds thanks to our assumption $\{\alpha\}^{n}-n\{\alpha\}^{n-1} .\{\beta\}>0$.

The obvious corollary of Lemma 4.16 is the following inequality:

$$
R A^{2}-\left(n s_{0}-1+R\right) A+(n-1) s_{0}<0 .
$$

- Monotonicity of $g:\left[1, \frac{R}{n}\right] \rightarrow \mathbb{R}$. Picking up where we left off in (51), we get the equivalence:

$$
g^{\prime}(t) \geq 0 \Longleftrightarrow t \geq s_{0} \frac{A-s_{0}+(n-R)(1-A)}{R A^{2}-\left(n s_{0}-1+R\right) A+(n-1) s_{0}} .
$$

Lemma 4.17 The following inequalities hold:

$$
\text { (a) } A-s_{0}+(n-R)(1-A)<0 \quad \text { and } \quad(b) 1>s_{0} \frac{A-s_{0}+(n-R)(1-A)}{R A^{2}-\left(n s_{0}-1+R\right) A+(n-1) s_{0}} \text {. }
$$

Proof. (a) We have: $A-s_{0}+(n-R)(1-A)=\left(1-s_{0}\right)\left(1-\frac{n}{R}\right)+\frac{n}{R}\left(1-s_{0}\right)(n-R)=\frac{\left(1-s_{0}\right)(n-R)(n-1)}{R}$ and the last expression is negative since $n-R<0$ while $1-s_{0}>0$ and $n-1>0$.

(b) Thanks to (56), inequality $(b)$ in (58) is equivalent to

$$
\begin{aligned}
& R A^{2}-\left(n s_{0}-1+R\right) A+(n-1) s_{0}<s_{0}\left[A-s_{0}+(n-R)(1-A)\right] \Longleftrightarrow \\
& R A^{2}-\left(R s_{0}+R+s_{0}-1\right) A+s_{0}\left(s_{0}+R-1\right)<0 .
\end{aligned}
$$

The discriminant of the l.h.s. in (59), viewed as a $2^{\text {nd }}$ degree polynomial in $A$, is $\Delta^{\prime \prime}=(R-1)^{2}(1-$ $\left.s_{0}\right)^{2}$, so the l.h.s. of (59) vanishes at

$A_{3}=\frac{R\left(s_{0}+1\right)+s_{0}-1-(R-1)\left(1-s_{0}\right)}{2 R}=s_{0} \quad$ and $\quad A_{4}=1-\frac{1-s_{0}}{R}, \quad$ where clearly $A_{3}<A_{4}$.

Thus, inequality (59) is equivalent to $s_{0}<A<1-\frac{1-s_{0}}{R}$. We have seen in Lemma 4.14 that $A>s_{0}$. On the other hand, proving $A<1-\frac{1-s_{0}}{R}$ amounts to proving

$$
\left.1-\frac{n}{R}\left(1-s_{0}\right)<1-\frac{1-s_{0}}{R} \Longleftrightarrow 1<n \quad \text { (since } 1-s_{0}>0 \text { and } R>0\right) \text {. }
$$

The last inequality being obvious, the proof of $(b)$ in (58) is complete. 
Conclusion 4.18 Inequality (57) holds strictly for every $t \geq 1$ thanks to part (b) of (58). So, in particular, $g^{\prime}(t)>0$ for all $t \in\left[1, \frac{R}{n}\right]$, i.e. the function $g:\left[1, \frac{R}{n}\right] \rightarrow \mathbb{R}$ is increasing.

Since $\operatorname{Vol}(\{\alpha-\beta\}) \geq g(t)$ for all $t \in\left[1, \frac{R}{n}\right]$ (cf. Lemma 4.14), the best lower bound for $\operatorname{Vol}(\{\alpha-\beta\})$ that we get through this method in the case when $s_{0}:=N^{(\beta)}(\alpha)<1$ is

$$
\operatorname{Vol}(\{\alpha-\beta\}) \geq g\left(\frac{R}{n}\right)=\left(\{\alpha\}^{n}-n\{\alpha\}^{n-1} \cdot\{\beta\}\right)\left(\frac{\{\alpha\}^{n}-n\{\alpha\}^{n-1} \cdot\{\beta\}}{\{\alpha\}^{n}-n s_{0}\{\alpha\}^{n-1} \cdot\{\beta\}}\right)^{n-1} .
$$

This proves Theorem 1.4. Note that this lower bound for the volume improves on the lower bound $g(1)$ (cf. (50)) obtained in (15).

\section{$5 \quad$ Intersection numbers}

In this section, we prove Theorem 1.3. We start by deriving analogues in bidegree $(p, p)$ with $p \geq 2$ of the inequalities established in $\S .3$. We will use the standard notion of positivity for $(q, q)$-forms whose definition is recalled at the beginning of the Appendix before Lemma 7.1.

Proposition 5.1 Let $X$ be a compact Kähler manifold with $\operatorname{dim}_{\mathbb{C}} X=n$ and let $\alpha, \beta$ be Kähler metrics on $X$. Then, for every $t \in[0,+\infty)$, every $p \in\{1, \ldots, n\}$ and every $C^{\infty}$ positive $(n-p, n-p)$ form $\Omega^{n-p, n-p} \geq 0$ on $X$ such that $\partial \bar{\partial} \Omega^{n-p, n-p}=0$, we have:

$$
\int_{X}\left(\alpha^{p}-t p \alpha^{p-1} \wedge \beta\right) \wedge \Omega^{n-p, n-p} \geq\left(1-t \frac{n}{R}\right) \int_{X} \alpha^{p} \wedge \Omega^{n-p, n-p},
$$

where, as usual, we let $R:=\frac{\{\alpha\}^{n}}{\{\alpha\}^{n-1} \cdot\{\beta\}}$. We also have:

$$
\int_{X}\left(\alpha^{p}-t^{p} \beta^{p}\right) \wedge \Omega^{n-p, n-p} \geq\left(1-t^{p} \frac{\left(\begin{array}{l}
n \\
p
\end{array}\right)}{R_{p}}\right) \int_{X} \alpha^{p} \wedge \Omega^{n-p, n-p},
$$

where we let $R_{p}:=\frac{\{\alpha\}^{n}}{\{\alpha\}^{n-p} \cdot\{\beta\}^{p}}$.

Proof. We may and will assume without loss of generality that $\Omega^{n-p, n-p}$ is strictly positive. Inequality (61) is equivalent to

$$
t \frac{n}{R} \int_{X} \alpha^{p} \wedge \Omega^{n-p, n-p} \geq t p \int_{X} \alpha^{p-1} \wedge \beta \wedge \Omega^{n-p, n-p},
$$

which, in turn, after the simplification of $t \geq 0$ and the unravelling of $R$, is equivalent to

$$
\frac{n}{p}\left(\int_{X} \alpha^{p} \wedge \Omega^{n-p, n-p}\right) \cdot\left(\int_{X} \alpha^{n-1} \wedge \beta\right) \geq\{\alpha\}^{n} \int_{X} \alpha^{p-1} \wedge \beta \wedge \Omega^{n-p, n-p} .
$$

This inequality can be proved using the method in the proof of Lemma 3.1, the pointwise inequality (82) proved in the Appendix and an approximate fixed point technique that we now describe. Here are the details. 


\section{Approximate fixed point technique}

We consider the following Monge-Ampère equation whose unique $C^{\infty}$ solution in the Kähler class $\{\alpha\}$ is denoted by $\widetilde{\alpha}:=\alpha+i \partial \bar{\partial} \varphi>0$ :

$$
\widetilde{\alpha}^{n}=\frac{\{\alpha\}^{n}}{\{\alpha\}^{p-1} \cdot\{\beta\} \cdot\left[\Omega^{n-p, n-p}\right]_{A}} \alpha^{p-1} \wedge \beta \wedge \Omega^{n-p, n-p} .
$$

By $\{\alpha\}^{p-1} \cdot\{\beta\} \cdot\left[\Omega^{n-p, n-p}\right]_{A}$ we mean the positive real number $\int_{X} \alpha^{p-1} \wedge \beta \wedge \Omega^{n-p, n-p}$ which clearly depends only on the Bott-Chern classes $\{\alpha\},\{\beta\} \in H^{1,1}(X, \mathbb{R})$ and on the Aeppli class $\left[\Omega^{n-p, n-p}\right]_{A} \in$ $H_{A}^{n-p, n-p}(X, \mathbb{R})$.

We will vary the form $\alpha$ on the r.h.s. of (64) in its Kähler class $\{\alpha\}$. Let $\mathcal{E}_{\alpha}:=\{T \in\{\alpha\} / T \geq 0\}$ be the set of $d$-closed positive $(1,1)$-currents in the Kähler class $\{\alpha\}$. Thus $\mathcal{E}_{\alpha}$ is a compact convex subset of the locally convex space $\mathcal{D}^{\prime 1}, 1(X, \mathbb{R})$ endowed with the weak topology of currents. (The compactness is a consequence of the existence of Gauduchon metrics and holds for any psef class $\{\alpha\}$ even if $X$ is not Kähler.) Fix an arbitrary Kähler metric $\omega$ in $\{\alpha\}$. For every $\varepsilon>0$, we associate with equation (64) the map:

$$
R_{\varepsilon}: \mathcal{E}_{\alpha} \rightarrow \mathcal{E}_{\alpha}, \quad R_{\varepsilon}(T)=\alpha_{T, \varepsilon},
$$

defined in three steps as follows. Let $T \in \mathcal{E}_{\alpha}$ be arbitrary.

(i) By the Blocki-Kolodziej version [BK07] for Kähler classes of Demailly's regularisation-ofcurrents theorem [Dem92, Theorem 1.1], there exist $C^{\infty} d$-closed $(1,1)$-forms $\omega_{\varepsilon} \in\{\alpha\}=\{T\}$ for $\varepsilon>0$ such that $\omega_{\varepsilon} \geq-\varepsilon \omega$ and $\omega_{\varepsilon} \rightarrow T$ in the weak topology of currents as $\varepsilon \rightarrow 0$. (The Kähler assumption on the class $\{\alpha\}$ crucially ensures that the possible negative part of $\omega_{\varepsilon}$ does not exceed $\varepsilon \omega$, see $[\mathrm{BK} 07]$.

Note that for every sequence of currents $T_{j} \in \mathcal{E}_{\alpha}$ converging weakly to a current $T \in \mathcal{E}_{\alpha}$ and for every fixed $\varepsilon>0$, the sequence of $C^{\infty}$ forms $\left(\omega_{j, \varepsilon}\right)_{j}$ (obtained by applying to each $T_{j}$ the BlockiKolodziej regularisation procedure just described producing a family $\omega_{j, \varepsilon} \rightarrow T_{j}$ as $\varepsilon \rightarrow 0$ ) converges in the $C^{\infty}$ topology to the $C^{\infty}$ form $\omega_{\varepsilon}$ (obtained by applying to $T$ the Blocki-Kolodziej regularisation procedure producing a family $\omega_{\varepsilon} \rightarrow T$ as $\left.\varepsilon \rightarrow 0\right)$. In other words, for every fixed $\varepsilon>0$, the map $\mathcal{E}_{\alpha} \ni T \mapsto \omega_{\varepsilon} \in C_{1,1}^{\infty}(X, \mathbb{C})$ is continuous if $\mathcal{E}_{\alpha}$ has been equipped with the weak topology of currents and the space of smooth $(1,1)$-forms has been given the $C^{\infty}$ topology.

To see this, it suffices to work locally with currents $T_{j}=i \partial \bar{\partial} \psi_{j} \geq 0$ and $T=i \partial \bar{\partial} \psi \geq 0$ for which the psh potentials have the property that $\psi_{j} \longrightarrow \psi$ in the $L^{1}$ topology as $j \rightarrow+\infty$, and to show that for every fixed $\varepsilon>0$ we have $i \partial \bar{\partial} \psi_{j} \star \rho_{\varepsilon} \longrightarrow i \partial \bar{\partial} \psi \star \rho_{\varepsilon}$ in the $C^{0}$ topology as $j \rightarrow+\infty$. (The convergence in the $C^{\infty}$ topology follows from this by taking derivatives.) Indeed, currents are regularised in [BK07] by convolution of their local potentials with regularising kernels $\rho_{\varepsilon}$. Since $i \partial \bar{\partial} \psi_{j} \star \rho_{\varepsilon}=\psi_{j} \star i \partial \bar{\partial} \rho_{\varepsilon}$ and $i \partial \bar{\partial} \psi \star \rho_{\varepsilon}=\psi \star i \partial \bar{\partial} \rho_{\varepsilon}$, we have to ensure, for every fixed $\varepsilon>0$, that

$$
\int_{U^{\prime}}\left(\psi_{j}-\psi\right)(y) u_{\varepsilon}(x-y) \underset{j \rightarrow+\infty}{\longrightarrow} 0 \quad \text { locally uniformly w.r.t. } x \in U^{\prime} \Subset U
$$

for every $C^{\infty}$ function $u_{\varepsilon}$ (which is an arbitrary coefficient of $i \partial \bar{\partial} \rho_{\varepsilon}$ in this case) defined on the open subset $U \subset X$ on which we work. This is clear from the $L^{1}$ convergence $\psi_{j} \longrightarrow \psi$ on $U$. 
(ii) Set $u_{T, \varepsilon}:=(1-\varepsilon) \omega_{\varepsilon}+\varepsilon \omega$. Thus $u_{T, \varepsilon}$ is a Kähler metric in the class $\{\alpha\}$ since it is $C^{\infty}$ and $u_{T, \varepsilon} \geq-(1-\varepsilon) \varepsilon \omega+\varepsilon \omega=\varepsilon^{2} \omega>0$. Moreover, $u_{T, \varepsilon} \rightarrow T$ in the weak topology of currents as $\varepsilon \rightarrow 0$.

(iii) Solve equation (64) with right-hand term defined by $u_{T, \varepsilon}$ instead of $\alpha$ :

$$
\alpha_{T, \varepsilon}^{n}=\frac{\{\alpha\}^{n}}{\{\alpha\}^{p-1} \cdot\{\beta\} \cdot\left[\Omega^{n-p, n-p}\right]_{A}} u_{T, \varepsilon}^{p-1} \wedge \beta \wedge \Omega^{n-p, n-p} .
$$

This means that we denote by $\alpha_{T, \varepsilon}$ the unique Kähler metric in the Kähler class $\{\alpha\}$ solving equation (66) whose existence is ensured by Yau's theorem [Yau78]. We put $R_{\varepsilon}(T):=\alpha_{T, \varepsilon}$. Thus, in particular, the image of $R_{\varepsilon}$ consists of (smooth) Kähler metrics in $\{\alpha\}$.

Now, $R_{\varepsilon}$ is a continuous self-map of the compact convex subset $\mathcal{E}_{\alpha}$ of the locally convex space $\mathcal{D}^{\prime 1,1}(X, \mathbb{R})$, so by the Schauder fixed point theorem, there exists a current $T_{\varepsilon} \in \mathcal{E}_{\alpha}$ such that $T_{\varepsilon}=R_{\varepsilon}\left(T_{\varepsilon}\right)=\alpha_{T_{\varepsilon}, \varepsilon}$. Since $\alpha_{T_{\varepsilon}, \varepsilon}:=\widetilde{\alpha}_{\varepsilon}$ is $C^{\infty}$, by construction, the fixed-point current $T_{\varepsilon}$ must be a $C^{\infty}$ form, so $T_{\varepsilon}=\widetilde{\alpha}_{\varepsilon}$ and $\omega_{\varepsilon} \geq \widetilde{\alpha}_{\varepsilon}-\delta_{\varepsilon} \omega$ for some $\delta_{\varepsilon} \downarrow 0$ when $\varepsilon \rightarrow 0$. (The last statement follows from the fact that $\omega_{\varepsilon}$ converges in the $C^{\infty}$ topology to $T$ if $T$ is $C^{\infty}$ - see the explanations under (3) below.) Hence $u_{T_{\varepsilon}, \varepsilon}=(1-\varepsilon) \omega_{\varepsilon}+\varepsilon \omega \geq(1-\varepsilon) \widetilde{\alpha}_{\varepsilon}+\left[\varepsilon-(1-\varepsilon) \delta_{\varepsilon}\right] \omega$. We put $\eta_{\varepsilon}:=\varepsilon-(1-\varepsilon) \delta_{\varepsilon}$, so $\eta_{\varepsilon} \rightarrow 0$ when $\varepsilon \rightarrow 0$.

To conclude, for every $\varepsilon>0$, we have got a Kähler metric $\widetilde{\alpha}_{\varepsilon}$ in the Kähler class $\{\alpha\}$ such that

$$
\begin{aligned}
\widetilde{\alpha}_{\varepsilon}^{n} & =\frac{\{\alpha\}^{n}}{\{\alpha\}^{p-1} \cdot\{\beta\} \cdot\left[\Omega^{n-p, n-p}\right]_{A}}\left[(1-\varepsilon) \omega_{\varepsilon}+\varepsilon \omega\right]^{p-1} \wedge \beta \wedge \Omega^{n-p, n-p} \\
& \geq(1-\varepsilon)^{p-1} \frac{\{\alpha\}^{n}}{\{\alpha\}^{p-1} \cdot\{\beta\} \cdot\left[\Omega^{n-p, n-p}\right]_{A}} \widetilde{\alpha}_{\varepsilon}^{p-1} \wedge \beta \wedge \Omega^{n-p, n-p}-O\left(\left|\eta_{\varepsilon}\right|\right),
\end{aligned}
$$

where $\omega$ is an arbitrary, fixed Kähler metric in the class $\{\alpha\}$ and $O\left(\left|\eta_{\varepsilon}\right|\right)$ is a quantity that converges to zero as $\varepsilon \rightarrow 0$. The Kähler metric $\widetilde{\alpha}_{\varepsilon}$ can be viewed as an approximate fixed point in the class $\{\alpha\}$ of equation (64).

Explanations. Here are a few additional comments on the choice of a continuous regularising operator $R_{\varepsilon}$ for every $\varepsilon>0$. We are indebted to J.-P. Demailly and to A. Zeriahi for many of the ensuing remarks that were left out of the first version of this paper.

(1) The existence of a continuous regularising operator is an easy consequence of the regularisation theorem (whatever version of it may be used, be it Demailly's regularisation of currents [Dem92, Theorem 1.1] or the Blocki-Kolodziej one [BK07] or any other one) applied to finitely many currents. The argument for this statement, which has been very kindly and effectively explained to the author by J.-P. Demailly, makes use of the compactness and convexity of $\mathcal{E}_{\alpha}$ in $\mathcal{D}^{\prime 1}, 1(X, \mathbb{R})$. Indeed, the cone of positive currents has a compact and metrisable, hence countable, base. For this reason, there are several different topologies that induce the same topology on this cone $(=$ the weak topology of currents). If we fix a smooth representative $\alpha$ of the class $\{\alpha\}$ (and $\alpha$ can be chosen to be a Kähler metric in this case, but this is irrelevant here), any current $T \in \mathcal{E}_{\alpha}$ can be written as $T=\alpha+i \partial \bar{\partial} \varphi \geq 0$ for a global quasi-psh (hence $L^{2}$ and indeed $L^{p}$ for every $p \in[1,+\infty)$ ) potential $\varphi$ that is unique up to a constant. We can equip the space of potentials $\{\varphi / i \partial \bar{\partial} \varphi \geq-\alpha\}$ with the topology induced by the $L^{2}$ Hilbert space topology, which is separable, hence has a countable orthonormal base. This topology induces on $\mathcal{E}_{\alpha}$ the weak topology of currents. 
Now, by compactness of $\mathcal{E}_{\alpha}$, for every $\varepsilon$, there is a finite covering of $\mathcal{E}_{\alpha}$ by open balls of radius $\varepsilon$. Let $T_{1}, \ldots, T_{N_{\varepsilon}} \in \mathcal{E}_{\alpha}$ be the centres of these balls and let $\mathcal{E}_{\alpha, \varepsilon}$ be the convex polyhedron generated by $T_{1}, \ldots, T_{N_{\varepsilon}}$. We can take $\varepsilon=1 / m$ for $m \in \mathbb{N}^{\star}$ and by convexity of $\mathcal{E}_{\alpha}$ we get

$$
\mathcal{E}_{\alpha}=\bigcup_{m=1}^{+\infty} \mathcal{E}_{\alpha, \frac{1}{m}}
$$

Thus, it suffices to regularise the finitely many currents $T_{1}, \ldots, T_{N_{\varepsilon}}$ and to extend the regularisation to all the currents $T \in \mathcal{E}_{\alpha, \varepsilon}$ by mere convex combinations. This clearly produces a continuous regularising operator.

(2) The main result of [BK07], namely that in a Kähler class positive currents can be regularised with only an $O(\varepsilon)$ loss of positivity (so, ultimately, with no loss at all, as explained above - hence the Kähler metrics in a given Kähler class are dense in the positive currents of that class) can also be obtained as an easy consequence of Demailly's regularisation theorem [Dem92]. The argument for this statement, which was very kindly explained to the author by A. Zeriahi, proceeds by first regularising by a mere cut-off operation. Indeed, let $T=\alpha+i \partial \bar{\partial} \varphi \geq 0$ be an arbitrary positive current in the Kähler class $\{\alpha\}$, where $\alpha>0$ is a Kähler metric in this class. For every $\varepsilon>0$, put $T_{\varepsilon}:=\alpha+i \partial \bar{\partial} \max \left(\varphi,-\frac{1}{\varepsilon}\right) \geq 0$. The current $T_{\varepsilon}$ is still positive since the maximum of any two $\alpha$-psh functions is still $\alpha$-psh when $\alpha$ is a Kähler metric ([GZ05, Proposition 2.3, (4)]). We have $\max \left(\varphi,-\frac{1}{\varepsilon}\right) \downarrow \varphi$ pointwise and $T_{\varepsilon} \rightarrow T$ weakly as $\varepsilon \rightarrow 0$. Moreover, the currents $T_{\varepsilon}$ have bounded potentials, so we can apply Demailly's regularisation theorem [Dem92] to each of them to write $T_{\varepsilon}$ as the weak limit of a sequence of $C^{\infty}$ forms $T_{\varepsilon, \delta} \in\{\alpha\}$ as $\delta \rightarrow 0$. Since all the Lelong numbers of $T_{\varepsilon}$ vanish (because the potential is bounded), Demailly's theorem [Dem92] ensures that only a loss of positivity of $O(\delta)$ is introduced by the regularisation process. Taking the diagonal sequence with $\varepsilon=\delta$, we get an approximation of the original current $T$ by $C^{\infty}$ forms in its class with only an $O(\varepsilon)$ loss of positivity.

The interest in the Blocki-Kolodziej regularisation procedure [BK07] lies in its giving a much simpler proof of the existence of a good regularisation of currents (which is by no means unique) for the special case of a Kähler class than Demailly's proof of the general case.

(3) The Blocki-Kolodziej regularisation [BK07] proceeds by convolution of the local potentials of the current $T$ with regularising kernels $\rho_{\varepsilon}$. This method produces a continuous regularising operator $R_{\varepsilon}$ for every $\varepsilon$. Moreover, if $T$ is a $C^{\infty}$ form in the class $\{\alpha\}$, the $C^{\infty}$ forms $T_{\varepsilon}$ obtained by regularising $T$ converge to $T$ in the $C^{\infty}$ topology as $\varepsilon \rightarrow 0$. This is because locally, if $\psi$ is a $C^{\infty}$ function defined on an open subset $\Omega \subset \mathbb{C}^{n}$ containing the origin, then all the derivatives of the convolutions $\rho_{\varepsilon} \star \psi$ converge uniformly on all the compact subsets $K \subset \Omega$ to the corresponding derivatives of $\psi$ and the (standard) patching procedure used in [BK07] does not destroy this property. On the other hand, Yau's theorem [Yau78] gives uniform estimates in all the $C^{l}$ norms of the solution of the Monge-Ampère equation in terms of the r.h.s. term of this equation. Putting these facts together, we get that the regularising operator $R_{\varepsilon}$ obtained by regularisation followed by an application of Yau's theorem is indeed continuous in the weak topology of currents and, moreover, $R_{\varepsilon}(T)$ converges in the $C^{\infty}$ topology to $T$ whenever $T \in \mathcal{E}_{\alpha}$ is $C^{\infty}$.

Use of the approximate fixed point

Let us fix any smooth volume form $d V>0$ on $X$. The l.h.s. term in (63) reads: 


$$
\begin{aligned}
& \frac{n}{p}\left(\int_{X} \alpha^{p} \wedge \Omega^{n-p, n-p}\right) \cdot\left(\int_{X} \alpha^{n-1} \wedge \beta\right)=\frac{n}{p}\left(\int_{X} \frac{\widetilde{\alpha}_{\varepsilon}^{p} \wedge \Omega^{n-p, n-p}}{d V} d V\right) \cdot\left(\int_{X} \frac{\widetilde{\alpha}_{\varepsilon}^{n-1} \wedge \beta}{\widetilde{\alpha}_{\varepsilon}^{n}} \frac{\widetilde{\alpha}_{\varepsilon}^{n}}{d V} d V\right) \\
& \stackrel{(a)}{\geq}\left[\int_{X}\left(\frac{n}{p} \frac{\widetilde{\alpha}_{\varepsilon}^{p} \wedge \Omega^{n-p, n-p}}{d V} \frac{\widetilde{\alpha}_{\varepsilon}^{n-1} \wedge \beta}{\widetilde{\alpha}_{\varepsilon}^{n}}\right)^{\frac{1}{2}}\left(\frac{\widetilde{\alpha}_{\varepsilon}^{n}}{d V}\right)^{\frac{1}{2}} d V\right]^{2} \stackrel{(b)}{\geq}\left[\int_{X}\left(\frac{\widetilde{\alpha}_{\varepsilon}^{p-1} \wedge \beta \wedge \Omega^{n-p, n-p}}{d V}\right)^{\frac{1}{2}}\left(\frac{\widetilde{\alpha}_{\varepsilon}^{n}}{d V}\right)^{\frac{1}{2}} d V\right]^{2} \\
& \stackrel{(c)}{\geq}(1-\varepsilon)^{p-1} \frac{\{\alpha\}^{n}}{\{\alpha\}^{p-1} \cdot\{\beta\} \cdot\left[\Omega^{n-p, n-p}\right]_{A}}\left[\int_{X}\left(\frac{\widetilde{\alpha}_{\varepsilon}^{p-1} \wedge \beta \wedge \Omega^{n-p, n-p}}{d V}\right)^{\frac{1}{2}}\left(\frac{\widetilde{\alpha}_{\varepsilon}^{p-1} \wedge \beta \wedge \Omega^{n-p, n-p}}{d V}\right)^{\frac{1}{2}} d V\right]^{2} \\
& -O\left(\left|\eta_{\varepsilon}\right|\right) \\
& \stackrel{(d)}{=}(1-\varepsilon)^{p-1} \frac{\{\alpha\}^{n}}{\{\alpha\}^{p-1} \cdot\{\beta\} \cdot\left[\Omega^{n-p, n-p}\right]_{A}}\left[\int_{X} \alpha^{p-1} \wedge \beta \wedge \Omega^{n-p, n-p}\right]^{2}-O\left(\left|\eta_{\varepsilon}\right|\right) \\
& =(1-\varepsilon)^{p-1}\{\alpha\}^{n} \int_{X} \alpha^{p-1} \wedge \beta \wedge \Omega^{n-p, n-p}-O\left(\left|\eta_{\varepsilon}\right|\right) \text {, }
\end{aligned}
$$

for every $\varepsilon>0$. Letting $\varepsilon \rightarrow 0$, we get the desired inequality (63) since $\eta_{\varepsilon} \rightarrow 0$. Inequality $(a)$ was an application of the Cauchy-Schwarz inequality, (b) was an application of the pointwise inequality (82) of Lemma 7.1 in the Appendix, (c) followed from (67), while identity $(d)$ followed from $\widetilde{\alpha}_{\varepsilon}$ belonging to the class $\{\alpha\}$. The proof of (61), which is equivalent to (63), is complete.

The proof of (62) runs along the same lines. Indeed, (62) is equivalent to

$$
t^{p} \frac{\left(\begin{array}{l}
n \\
p
\end{array}\right)}{R_{p}} \int_{X} \alpha^{p} \wedge \Omega^{n-p, n-p} \geq t^{p} \int_{X} \beta^{p} \wedge \Omega^{n-p, n-p},
$$

which, in turn, after the simplification of $t^{p} \geq 0$ and the unravelling of $R_{p}$, is equivalent to

$$
\left(\begin{array}{l}
n \\
p
\end{array}\right)\left(\int_{X} \alpha^{p} \wedge \Omega^{n-p, n-p}\right) \cdot\left(\int_{X} \alpha^{n-p} \wedge \beta^{p}\right) \geq\{\alpha\}^{n} \int_{X} \beta^{p} \wedge \Omega^{n-p, n-p} .
$$

The proof of (68) is almost identical to that of (63) spelt out above except for the replacement of equation (64) with the following Monge-Ampère equation:

$$
\widetilde{\alpha}^{n}=\frac{\{\alpha\}^{n}}{\{\beta\}^{p} \cdot\left[\Omega^{n-p, n-p}\right]_{A}} \beta^{p} \wedge \Omega^{n-p, n-p}
$$

and for the replacement of the pointwise inequality (82) with (81). Note that, since $\alpha$ does not feature on the r.h.s. of equation (69), the approximate fixed point technique is no longer necessary in this case. It suffices to work with the unique Kähler-metric solution $\widetilde{\alpha}$ of (69).

Remark 5.2 If an exact (rather than an approximate) fixed point for equation (64) had been sought, we would have needed to consider the following equation in which the Kähler-metric solution $\widetilde{\alpha} \in\{\alpha\}$ features on both sides:

$$
\widetilde{\alpha}^{n}=\frac{\{\alpha\}^{n}}{\{\alpha\}^{p-1} \cdot\{\beta\} \cdot\left[\Omega^{n-p, n-p}\right]_{A}} \widetilde{\alpha}^{p-1} \wedge \beta \wedge \Omega^{n-p, n-p} .
$$


Equations of this type, going back to Donaldson's J-flow and to work by Chen, admit a solution under a certain assumption on the class $\{\alpha\}$. See [FLM11] and the references therein for details. Our approximate fixed point technique does not require any particular assumption on $\{\alpha\}$.

We can now prove the main result of this section which subsumes Theorem 1.3.

Theorem 5.3 Let $X$ be a compact Kähler manifold with $\operatorname{dim}_{\mathbb{C}} X=n$ and let $\{\alpha\},\{\beta\}$ be Kähler classes such that $\{\alpha\}^{n}-n\{\alpha\}^{n-1} .\{\beta\}>0$. Then, for every $k \in\{1,2, \ldots, n\}$ and every smooth positive $(n-k, n-k)$-form $\Omega^{n-k, n-k} \geq 0$ such that $\partial \bar{\partial} \Omega^{n-k, n-k}=0$, the following inequalities hold:

$$
\begin{aligned}
&\left\{\alpha^{k}-\beta^{k}\right\} \cdot\left[\Omega^{n-k, n-k}\right]_{A} \stackrel{\left(I_{k}\right)}{\geq}\{\alpha-\beta\}^{k} \cdot\left[\Omega^{n-k, n-k}\right]_{A} \underset{\stackrel{\left(I I_{k}\right)}{\geq}}{\geq}\left\{\alpha^{k}-k \alpha^{k-1} \wedge \beta\right\} \cdot\left[\Omega^{n-k, n-k}\right]_{A} \\
& \stackrel{\left(I I I_{k}\right)}{\geq}\left(1-\frac{n}{R}\right)\{\alpha\}^{k} \cdot\left[\Omega^{n-k, n-k}\right]_{A} \geq 0
\end{aligned}
$$

where, as usual, $R:=\frac{\{\alpha\}^{n}}{\{\alpha\}^{n-1} \cdot\{\beta\}}$. (Thus $R>n$ by assumption.) In particular, $\left(I I_{n}\right)$ and $\left(I I I_{n}\right)$ read:

$$
\{\alpha-\beta\}^{n} \geq\{\alpha\}^{n}-n\{\alpha\}^{n-1} \cdot\{\beta\}=\left(1-\frac{n}{R}\right)\{\alpha\}^{n}>0 .
$$

Proof. We may and will assume without loss of generality that $\Omega^{n-k, n-k}$ is strictly positive.

Inequality $\left(I I I_{k}\right)$ is nothing but (61) for $t=1$ and $p=k$.

We will now prove $\left(I I_{k}\right)$ by induction on $k \in\{1, \ldots, n\}$. Let us fix Kähler metrics $\alpha, \beta$ in the classes $\{\alpha\}$, resp. $\{\beta\}$. For $k=1,\left(I I_{1}\right)$ is obviously an identity. Now, proving $\left(I I_{k}\right)$ for an arbitrary $k$ amounts to proving that the quantity

$$
S_{k}:=\int_{X}\left((\alpha-\beta)^{k}-\alpha^{k}+k \alpha^{k-1} \wedge \beta\right) \wedge \Omega^{n-k, n-k}
$$

is non-negative. To this end, we first prove the identity:

$$
S_{k}=\sum_{l=1}^{k-1} l \int_{X}(\alpha-\beta)^{k-l-1} \wedge \alpha^{l-1} \wedge \beta^{2} \wedge \Omega^{n-k, n-k}, \quad k=1, \ldots, n .
$$

This follows immediately by writing the next pointwise identities: 


$$
\begin{aligned}
& (\alpha-\beta)^{k}-\alpha^{k}+k \alpha^{k-1} \wedge \beta=-\beta \wedge \sum_{l=1}^{k}(\alpha-\beta)^{k-l} \wedge \alpha^{l-1}+k \alpha^{k-1} \wedge \beta \\
= & \sum_{l=1}^{k-1} \alpha^{l-1} \wedge \beta \wedge\left(\alpha^{k-l}-(\alpha-\beta)^{k-l}\right)=\sum_{l=1}^{k-1} \alpha^{l-1} \wedge \beta^{2} \wedge \sum_{r=0}^{k-l-1} \alpha^{k-l-1-r} \wedge(\alpha-\beta)^{r} \\
= & \sum_{l=1}^{k-1} \sum_{r=0}^{k-l-1} \alpha^{k-r-2} \wedge \beta^{2} \wedge(\alpha-\beta)^{r} \\
= & \sum_{r=0}^{k-2} \alpha^{k-r-2} \wedge \beta^{2} \wedge(\alpha-\beta)^{r}+\cdots+\sum_{r=0}^{k-l-1} \alpha^{k-r-2} \wedge \beta^{2} \wedge(\alpha-\beta)^{r}+\cdots+\alpha^{k-2} \wedge \beta^{2} \\
= & \beta^{2} \wedge(\alpha-\beta)^{k-2}+2 \alpha \wedge \beta^{2} \wedge(\alpha-\beta)^{k-3}+\cdots+l \alpha^{l-1} \wedge \beta^{2} \wedge(\alpha-\beta)^{k-l-1}+\cdots+(k-1) \alpha^{k-2} \wedge \beta^{2} \\
= & \sum_{l=1}^{k-1} l(\alpha-\beta)^{k-l-1} \wedge \alpha^{l-1} \wedge \beta^{2} .
\end{aligned}
$$

This clearly proves (73).

Now we can run the induction on $k \in\{1, \ldots, n\}$ to prove $\left(I I_{k}\right)$. Suppose that $\left(I I_{1}\right), \ldots,\left(I I_{k-1}\right)$ have been proved. Combining them with $\left(I I I_{k}\right)$ that was proved in (61) for all $k \in\{1, \ldots, n\}$, we deduce that the classes $\{\alpha-\beta\}^{k-r}$ are positive in the following sense:

$$
\{\alpha-\beta\}^{k-r} \cdot\left[\Omega^{n-k+r, n-k+r}\right]_{A} \geq 0
$$

for all $r \in\{1, \ldots, k\}$ and for all $C^{\infty}$ strictly positive $(n-k+r, n-k+r)$-forms $\Omega^{n-k+r, n-k+r}>0$ such that $\partial \bar{\partial} \Omega^{n-k+r, n-k+r}=0$.

Choosing forms of the shape $\Omega^{n-k+r, n-k+r}:=\alpha^{r-2} \wedge \beta^{2} \wedge \Omega^{n-k, n-k}$ with $\Omega^{n-k, n-k}>0$ of bidegree $(n-k, n-k)$ satisfying $\partial \bar{\partial} \Omega^{n-k, n-k}=0$, we get:

$$
\{\alpha-\beta\}^{k-r} \cdot\{\alpha\}^{r-2} \cdot\{\beta\}^{2} \cdot\left[\Omega^{n-k, n-k}\right]_{A} \geq 0, \quad r \in\{2, \ldots, k\} .
$$

Setting $r:=l+1$, this translates to

$$
\int_{X}(\alpha-\beta)^{k-l-1} \wedge \alpha^{l-1} \wedge \beta^{2} \wedge \Omega^{n-k, n-k} \geq 0, \quad l \in\{1, \ldots, k-1\},
$$

which means precisely that all the terms in the sum expressing $S_{k}$ in (73) are non-negative. Hence, $S_{k} \geq 0$, which proves $\left(I I_{k}\right)$ (see 72 ).

Let us now prove $\left(I_{k}\right)$ as a consequence of $\left(I I_{k}\right)$ and $\left(I I I_{k}\right)$. For every $k \in\{1, \ldots, n\}$, the following pointwise identities are obvious:

$$
\begin{aligned}
\alpha^{k}-\beta^{k}-(\alpha-\beta)^{k} & =\beta \wedge \sum_{l=0}^{k-1} \alpha^{k-l-1} \wedge(\alpha-\beta)^{l}-\beta^{k}=\beta \wedge\left(\alpha^{k-1}-\beta^{k-1}+\sum_{l=1}^{k-1} \alpha^{k-l-1} \wedge(\alpha-\beta)^{l}\right) \\
& =\beta \wedge\left((\alpha-\beta) \wedge \sum_{r=0}^{k-2} \alpha^{k-r-2} \wedge \beta^{r}+\sum_{l=1}^{k-1} \alpha^{k-l-1} \wedge(\alpha-\beta)^{l}\right) .
\end{aligned}
$$


Hence, for every smooth $(n-k, n-k)$-form $\Omega^{n-k, n-k} \geq 0$ such that $\partial \bar{\partial} \Omega^{n-k, n-k}=0$, we have:

$$
\begin{aligned}
\left(\left\{\alpha^{k}-\beta^{k}\right\}-\{\alpha-\beta\}^{k}\right) \cdot\left[\Omega^{n-k, n-k}\right]_{A} & =\sum_{r=0}^{k-2} \int_{X}(\alpha-\beta) \wedge \alpha^{k-r-2} \wedge \beta^{r+1} \wedge \Omega^{n-k, n-k} \\
& +\sum_{l=1}^{k-1} \int_{X}(\alpha-\beta)^{l} \wedge \alpha^{k-l-1} \wedge \beta \wedge \Omega^{n-k, n-k} \\
& =\sum_{r=0}^{k-2}\{\alpha-\beta\} \cdot\left[\Omega_{r}^{n-1, n-1}\right]_{A}+\sum_{l=1}^{k-1}\{\alpha-\beta\}^{l} \cdot\left[\Gamma_{l}^{n-l, n-l}\right]_{A} \\
& \geq 0
\end{aligned}
$$

where we have put $\Omega_{r}^{n-1, n-1}:=\alpha^{k-r-2} \wedge \beta^{r+1} \wedge \Omega^{n-k, n-k}$ and $\Gamma_{l}^{n-l, n-l}:=\alpha^{k-l-1} \wedge \beta \wedge \Omega^{n-k, n-k}$. It is clear that $\Omega_{r}^{n-1, n-1}$ and $\Gamma_{l}^{n-l, n-l}$ are positive $\partial \bar{\partial}$-closed forms of bidegree $(n-1, n-1)$, resp. $(n-l, n-l)$, so the last inequality follows from the combination of $\left(I I_{k}\right)$ and $\left(I I I_{k}\right)$. Thus $\left(I_{k}\right)$ is proved.

We immediately get the following consequence of Theorem 5.3 which is the analogue of Theorem 3.5 in bidegree $(k, k)$ for an arbitrary $k$.

Corollary 5.4 Let $X$ be a compact Kähler manifold with $\operatorname{dim}_{\mathbb{C}} X=n$ and let $\alpha, \beta>0$ be Kähler metrics such that $\{\alpha\}^{n}-n\{\alpha\}^{n-1} \cdot\{\beta\}>0$. Then, for every $k \in\{1,2, \ldots, n\}$, there exist closed positive $(k, k)$-currents $U_{k} \in\left\{\alpha^{k}-\beta^{k}\right\}$ and $S_{k} \in\left\{(\alpha-\beta)^{k}\right\}$ such that

$$
U_{k} \geq\left(1-\frac{n}{R}\right) \alpha^{k} \quad \text { and } \quad S_{k} \geq\left(1-\frac{n}{R}\right) \alpha^{k}
$$

on $X$, where, as usual, we let $R:=\frac{\{\alpha\}^{n}}{\{\alpha\}^{n-1} \cdot\{\beta\}}$. (So $R>n$ by assumption.)

Proof. This follows immediately from Theorem 5.3 by using the analogue of Lamari's positivity criterion [Lam99, Lemme 3.3] in bidegree $(k, k)$ for every $k$.

\section{A conjecture in the non-Kähler context}

Let $X$ be a compact complex manifold with $\operatorname{dim}_{\mathbb{C}} X=n$. It is standard that if $X$ is of class $\mathcal{C}$, then $X$ is both balanced (i.e. it admits a balanced metric: a Hermitian metric $\omega$ such that $d \omega^{n-1}=0$ ) by [AB93, Corollary 4.5] and a $\partial \bar{\partial}$-manifold (i.e. the $\partial \bar{\partial}$-lemma holds on $X$ ). On the other hand, there are a great deal of examples of balanced manifolds that are not $\partial \bar{\partial}$-manifolds (e.g. the Iwasawa manifold), but it is still an open problem to find out whether or not every $\partial \bar{\partial}$-manifold admits a balanced metric. To the author's knowledge, all the examples of $\partial \bar{\partial}$-manifolds known so far are also balanced. We now briefly indicate how a generalised version of Demailly's Transcendental Morse Inequalities Conjecture for a difference of two nef classes might answer a stronger version of this question. The main idea is borrowed from Toma's work [Tom10] in the projective setting and was also exploited in [CRS14] in the Kähler setting. 
It is standard that the canonical linear map induced in cohomology by the identity:

$$
I_{n-1}: H_{B C}^{n-1, n-1}(X, \mathbb{C}) \rightarrow H_{A}^{n-1, n-1}(X, \mathbb{C}), \quad[\Omega]_{B C} \mapsto[\Omega]_{A},
$$

is well defined on every $X$, but it is neither injective, nor surjective in general. Moreover, the balanced cone of $X$ consisting of Bott-Chern cohomology classes of bidegree $(n-1, n-1)$ representable by balanced metrics $\omega^{n-1}$ :

$$
\mathcal{B}_{X}=\left\{\left[\omega^{n-1}\right]_{B C} / \omega>0, C^{\infty}(1,1) \text {-form such that } d \omega^{n-1}=0 \text { on } X\right\} \subset H_{B C}^{n-1, n-1}(X, \mathbb{R}),
$$

maps under $I_{n-1}$ to a subset of the Gauduchon cone of $X$ (introduced in [Pop13]) consisting of Aeppli cohomology classes of bidegree $(n-1, n-1)$ representable by Gauduchon metrics $\omega^{n-1}$ :

$$
\mathcal{G}_{X}=\left\{\left[\omega^{n-1}\right]_{A} / \omega>0, C^{\infty}(1,1) \text {-form such that } \partial \bar{\partial} \omega^{n-1}=0 \text { on } X\right\} \subset H_{A}^{n-1, n-1}(X, \mathbb{R}) .
$$

Clearly, the inclusion $I_{n-1}\left(\mathcal{B}_{X}\right) \subset \mathcal{G}_{X}$ is strict in general. So is the inclusion $I_{n-1}\left(\overline{\mathcal{B}_{X}}\right) \subset \overline{\mathcal{G}}_{X}$ involving the closures of these two open convex cones.

Now, if $X$ is a $\partial \bar{\partial}$-manifold, $I_{n-1}$ is an isomorphism of the vector spaces $H_{B C}^{n-1, n-1}(X, \mathbb{C})$ and $H_{A}^{n-1, n-1}(X, \mathbb{C})$, as is well known. It is tempting to make the following

Conjecture 6.1 If $X$ is a compact $\partial \bar{\partial}$-manifold of dimension $n$, then $I_{n-1}\left(\overline{\mathcal{B}_{X}}\right)=\overline{\mathcal{G}}_{X}$.

If proved to hold, this conjecture would imply that every $\partial \bar{\partial}$-manifold is actually balanced since the Gauduchon cone is never empty (due to the existence of Gauduchon metrics by [Gau77]), so the balanced cone would also have to be non-empty in this case. Moreover, a positive answer to this conjecture would have far-reaching implications for a possible future non-Kähler mirror symmetry theory since it would remove the ambiguity of choice between the balanced and the Gauduchon cones on $\partial \bar{\partial}$-manifolds. These two cones would be canonically equivalent on $\partial \bar{\partial}$-manifolds in this event.

One piece of evidence supporting Conjecture 6.1 is that it holds on every class $\mathcal{C}$ manifold $X$ if the whole of Demailly's Transcendental Morse Inequalities Conjecture for a difference of two nef classes is confirmed when $X$ is Kähler. This is the gist of the observations made in [Tom10] and in [CRS14] alluded to above. Indeed, if $X$ is of class $\mathcal{C}$, we may assume without loss of generality that $X$ is actually compact Kähler. As proved in [BDPP13], a complete positive answer to Conjecture 1.1 would imply that the pseudo-effective cone $\mathcal{E}_{X} \subset H^{1,1}(X, \mathbb{R})$ of classes of $d$-closed positive $(1,1)$ currents $T$ is the dual of the cone $\mathcal{M}_{X} \subset H^{n-1, n-1}(X, \mathbb{R})$ of movable classes (i.e. the closure of the cone generated by classes of currents of the shape $\mu_{\star}\left(\widetilde{\omega}_{1} \wedge \cdots \wedge \widetilde{\omega}_{n-1}\right)$, where $\mu: \widetilde{X} \rightarrow X$ is any modification of compact Kähler manifolds and the $\widetilde{\omega}_{j}$ are any Kähler metrics on $\widetilde{X}-$ see [BDPP13, Definition 1.3]). Since on $\partial \bar{\partial}$-manifolds (hence, in particular, on compact Kähler ones) the BottChern, Dolbeault and Aeppli cohomologies are canonically equivalent, it is irrelevant in which of these cohomologies the groups $H^{1,1}(X, \mathbb{R})$ and $H^{n-1, n-1}(X, \mathbb{R})$ are considered.

The closure $\overline{\mathcal{G}}_{X} \subset H^{n-1, n-1}(X, \mathbb{R})$ of the Gauduchon cone is dual to the pseudo-effective cone $\mathcal{E}_{X} \subset H^{1,1}(X, \mathbb{R})$ by Lamari's positivity criterion (Lemma 1.5), while the same kind of argument (i.e. duality and Hahn-Banach) going back to Sullivan shows that the closure $\overline{\mathcal{B}}_{X} \subset H^{n-1, n-1}(X, \mathbb{R})$ of the balanced cone is dual to the cone 


$$
\mathcal{S}_{X}=\left\{[T]_{A} / T \geq 0, T \text { is a }(1,1) \text { - current such that } \partial \bar{\partial} T=0 \text { on } X\right\} \subset H_{A}^{1,1}(X, \mathbb{R})
$$

Note that $\mathcal{S}_{X}$ is closed if $X$ admits a balanced metric $\omega^{n-1}$ (against which the masses of positive $\partial \bar{\partial}$-closed $(1,1)$-currents $T$ can be considered), hence so is it when $X$ is Kähler. Thus, by duality, the identity $I_{n-1}\left(\overline{\mathcal{B}_{X}}\right)=\overline{\mathcal{G}}_{X}$ is equivalent to $I_{1}\left(\mathcal{E}_{X}\right)=\mathcal{S}_{X}$, where $I_{1}$ is the canonical linear map induced in cohomology by the identity:

$$
I_{1}: H_{B C}^{1,1}(X, \mathbb{C}) \rightarrow H_{A}^{1,1}(X, \mathbb{C}), \quad[\gamma]_{B C} \mapsto[\gamma]_{A}
$$

In general, $I_{1}$ is neither injective, nor surjective, but it is an isomorphism when $X$ is a $\partial \bar{\partial}$-manifold.

With these facts understood, the identity $I_{1}\left(\mathcal{E}_{X}\right)=\mathcal{S}_{X}$ can be proved when $X$ is Kähler (provided that Conjecture 1.1 can be solved in the affirmative) as explained in [CRS14, Proposition 2.5] by an argument generalising to transcendental classes an earlier argument from [Tom10] that we now recall for the reader's convenience.

The inclusion $I_{1}\left(\mathcal{E}_{X}\right) \subset \mathcal{S}_{X}$ is obvious. To prove the reverse inclusion, let $[T]_{A} \in \mathcal{S}_{X}$, i.e. $T \geq 0$ is a $(1,1)$-current such that $\partial \bar{\partial} T=0$. Since $I_{1}$ is an isomorphism, there exists a unique class $[\gamma]_{B C} \in H_{B C}^{1,1}(X, \mathbb{R})$ such that $I_{1}\left([\gamma]_{B C}\right)=[T]_{A}$. This means that $[\gamma]_{A}=[T]_{A}$. We will show that $[\gamma]_{B C} \in \mathcal{E}_{X}$. If the $[\mathrm{BDPP} 13]$ conjecture (predicated on Conjecture 1.1) predicting that $\mathcal{E}_{X}$ is dual to $\mathcal{M}_{X}$ is confirmed, showing that $[\gamma]_{B C} \in \mathcal{E}_{X}$ amounts to showing that

$$
[\gamma]_{B C} \cdot\left[\mu_{\star}\left(\widetilde{\omega}_{1} \wedge \cdots \wedge \widetilde{\omega}_{n-1}\right)\right]_{A} \geq 0
$$

for all modifications $\mu: \widetilde{X} \rightarrow X$ and all Kähler metrics $\widetilde{\omega}_{j}$ on $\widetilde{X}$. On the other hand, Alessandrini and Bassanelli proved in [AB96, Theorem 5.6] the existence and uniqueness of the inverse image under proper modifications $\mu: \widetilde{X} \rightarrow X$ of arbitrary complex manifolds of any positive $\partial \bar{\partial}$-closed $(1,1)$-current $T \geq 0$ in such a way that the Aeppli cohomology class $[T]_{A}$ is preserved:

$\exists !(1,1)-$ current $\mu^{\star} T \geq 0$ on $\tilde{X}$ such that $\partial \bar{\partial}\left(\mu^{\star} T\right)=0, \quad\left[\mu^{\star} T\right]_{A}=\mu^{\star}\left([T]_{A}\right)$ and $\mu_{\star}\left(\mu^{\star} T\right)=T$.

(Note that the inverse image $\mu^{\star}\left([T]_{A}\right)$ of any Aeppli class is trivially well defined by taking smooth representatives of the class and pulling them back. Indeed, $\partial \bar{\partial}$-closedness is preserved, while pullbacks of Aeppli-cohomologous smooth forms are trivially seen to be Aeppli-cohomologous.) Using this key ingredient from [AB96], we get:

$$
\begin{aligned}
{[\gamma]_{B C} \cdot\left[\mu_{\star}\left(\widetilde{\omega}_{1} \wedge \cdots \wedge \widetilde{\omega}_{n-1}\right)\right]_{A} } & =\int_{X} \gamma \wedge \mu_{\star}\left(\widetilde{\omega}_{1} \wedge \cdots \wedge \widetilde{\omega}_{n-1}\right)=\int_{\widetilde{X}}\left(\mu^{\star} \gamma\right) \wedge\left(\widetilde{\omega}_{1} \wedge \cdots \wedge \widetilde{\omega}_{n-1}\right) \\
& =\left[\mu^{\star} \gamma\right]_{A} \cdot\left[\widetilde{\omega}_{1} \wedge \cdots \wedge \widetilde{\omega}_{n-1}\right]_{B C}=\int_{\widetilde{X}}\left(\mu^{\star} T\right) \wedge\left(\widetilde{\omega}_{1} \wedge \cdots \wedge \widetilde{\omega}_{n-1}\right) \geq 0
\end{aligned}
$$

which proves (77). Note that $\gamma$ and $\mu^{\star} \gamma$ have no sign, so the key point has been the replacement in the integral over $\widetilde{X}$ of $\mu^{\star} \gamma$ by $\mu^{\star} T \geq 0$ which was made possible by $\widetilde{\omega}_{1} \wedge \cdots \wedge \widetilde{\omega}_{n-1}$ being $d$-closed 
(so we could switch the roles of the Bott-Chern and the Aeppli cohomologies) and by the identity $\left[\mu^{\star} \gamma\right]_{A}=\left[\mu^{\star} T\right]_{A}$ following from $[\gamma]_{A}=[T]_{A}$ (see above) and from $\left[\mu^{\star} T\right]_{A}=\mu^{\star}\left([T]_{A}\right)$.

The techniques employed in this paper do not seem to be using the full force of the Kähler assumption on $X$ and many of the arguments are valid in a more general context. This is part of the justification for proposing Conjecture 6.1.

\section{Appendix: Hovanskii-Teissier-type inequalities}

In this section, we prove the pointwise inequalities for Hermitian metrics that were used in earlier sections. They generalise the inequality in [Pop14, Lemma 3.1].

For the sake of enhanced flexibility, we shall deal with positive $(q, q)$-forms that are not necessarily the $q^{\text {th }}$ power of a positive $(1,1)$-form. Given any $q \in\{0, \ldots, n\}$ and any $C^{\infty}$ real $(q, q)$-form $\Omega^{q, q}$ on $X$, we make use of the standard notion of (weak) positivity (see e.g. [Dem97, III.1.1]): $\Omega^{q, q}$ is said to be positive (resp. strictly positive) if for any $(1,0)$-forms $\alpha_{1}, \ldots \alpha_{n-q}$, the $(n, n)$-form $\Omega^{q, q} \wedge i \alpha_{1} \wedge \bar{\alpha}_{1} \wedge \cdots \wedge i \alpha_{n-q} \wedge \bar{\alpha}_{n-q}$ is non-negative (resp. positive). We write $\Omega^{q, q} \geq 0$ (resp. $\left.\Omega^{q, q}>0\right)$ in this case. If, in local holomorphic coordinates $z_{1}, \ldots, z_{n}$, we write

$$
\frac{\Omega^{q, q}}{q !}=\sum_{|L|=|R|=q} \Omega_{L \bar{R}} i d z_{L} \wedge d \bar{z}_{R},
$$

then it is clear by considering $\Omega^{q, q} \wedge i d z_{s_{1}} \wedge d \bar{z}_{s_{1}} \wedge \cdots \wedge i d z_{s_{n-q}} \wedge d \bar{z}_{s_{n-q}}$ that

$$
\Omega^{q, q} \geq 0 \text { implies } \Omega_{L \bar{L}} \geq 0 \text { for all } L \text { with }|L|=q .
$$

(We have used the usual notation: $L$ and $R$ stand for ordered multi-indices $L=\left(1 \leq l_{1}<\cdots<l_{q} \leq\right.$ $n)$, resp. $R=\left(1 \leq r_{1}<\cdots<r_{q} \leq n\right)$ of length $q$ and $i d z_{L} \wedge d \bar{z}_{R}:=i d z_{l_{1}} \wedge d \bar{z}_{r_{1}} \wedge \cdots \wedge i d z_{l_{q}} \wedge d \bar{z}_{r_{q}}$.)

In the special case when $\Omega^{q, q}=\gamma^{q}$ for some positive definite smooth $(1,1)$-form (= Hermitian metric) $\gamma$ on $X$, if we write

$$
\gamma=\sum_{j, k=1}^{n} \gamma_{j \bar{k}} i d z_{j} \wedge d \bar{z}_{k}
$$

then Sylvester's criterion ensures that $M_{L \bar{L}}(\gamma)>0$ for all multi-indices $L \subset\{1, \ldots, n\}$ of any length $l \in\{1, \ldots, n\}$. (For any multi-indices $L, K \subset\{1, \ldots, n\}$ of equal lengths, $M_{K \bar{L}}(\gamma)$ denotes the minor of the matrix $\left(\gamma_{j \bar{k}}\right)_{j, k}$ corresponding to the rows with index in $K$ and the columns with index in $L$.) Clearly, $M_{L \bar{L}}(\gamma)=\Omega_{L \bar{L}}$ for all $L$ with $|L|=q$.

Lemma 7.1 Let $\alpha, \beta$ be arbitrary Hermitian metrics on a complex manifold $X$ with dim $_{\mathbb{C}} X=n$. The following pointwise inequalities hold for every $p \in\{1, \ldots, n\}$ and for every smooth form $\Omega^{n-p, n-p} \geq 0$ of bidegree $(n-p, n-p)$ on $X$ :

$$
\left(\begin{array}{l}
n \\
p
\end{array}\right) \frac{\alpha^{n-p} \wedge \beta^{p}}{\alpha^{n}} \cdot \frac{\alpha^{p} \wedge \Omega^{n-p, n-p}}{\alpha^{n}} \geq \frac{\beta^{p} \wedge \Omega^{n-p, n-p}}{\alpha^{n}}
$$

and 


$$
\frac{n}{p} \frac{\alpha^{n-1} \wedge \beta}{\alpha^{n}} \cdot \frac{\alpha^{p} \wedge \Omega^{n-p, n-p}}{\alpha^{n}} \geq \frac{\alpha^{p-1} \wedge \beta \wedge \Omega^{n-p, n-p}}{\alpha^{n}}
$$

Proof. Let us first prove (81). The special case when $p=1$ and $\Omega^{n-1, n-1}=\gamma^{n-1}$ for some $(1,1)$-form $\gamma>0$ was proved in [Pop14, Lemma 3.1]. We fix any point $x \in X$ and choose local coordinates $z_{1}, \ldots, z_{n}$ about $x$ such that

$$
\alpha(x)=\sum_{j=1}^{n} i d z_{j} \wedge d \bar{z}_{j} \quad \text { and } \quad \beta(x)=\sum_{j=1}^{n} \beta_{j} i d z_{j} \wedge d \bar{z}_{j}
$$

Thus $\beta_{j}>0$ for all $j$. At $x$ we get: $\frac{\beta^{p}}{p !}=\sum_{j_{1}<\cdots<j_{p}} \beta_{j_{1}} \ldots \beta_{j_{p}} \bigwedge_{l \in\left\{j_{1}, \ldots, j_{p}\right\}}\left(i d z_{l} \wedge d \bar{z}_{l}\right)$, hence

$$
\frac{\alpha^{n-p} \wedge \beta^{p}}{\alpha^{n}}=\frac{1}{\left(\begin{array}{l}
n \\
p
\end{array}\right)} \sum_{j_{1}<\cdots<j_{p}} \beta_{j_{1}} \ldots \beta_{j_{p}}=\frac{\beta_{1} \ldots \beta_{n}}{\left(\begin{array}{l}
n \\
p
\end{array}\right)}\left(\sum_{|K|=n-p} \frac{1}{\beta_{K}}\right) \text { at } x,
$$

where $\beta_{K}:=\beta_{k_{1}} \ldots \beta_{k_{n-p}}$ whenever $K=\left(1 \leq k_{1}<\ldots k_{n-p} \leq n\right)$. On the other hand, using (78) with $q=n-p$, we get at $x$ :

$$
\frac{\alpha^{p} \wedge \Omega^{n-p, n-p}}{\alpha^{n}}=\frac{1}{\left(\begin{array}{l}
n \\
p
\end{array}\right)} \sum_{|L|=n-p} \Omega_{L \bar{L}} \text { and } \frac{\beta^{p} \wedge \Omega^{n-p, n-p}}{\alpha^{n}}=\frac{\beta_{1} \ldots \beta_{n}}{\left(\begin{array}{l}
n \\
p
\end{array}\right)} \sum_{|L|=n-p} \frac{\Omega_{L \bar{L}}}{\beta_{L}} .
$$

Thus, inequality (81) at $x$ is equivalent to:

$$
\left(\sum_{|L|=n-p} \Omega_{L \bar{L}}\right) \frac{\beta_{1} \ldots \beta_{n}}{\left(\begin{array}{l}
n \\
p
\end{array}\right)}\left(\sum_{|K|=n-p} \frac{1}{\beta_{K}}\right) \geq \frac{\beta_{1} \ldots \beta_{n}}{\left(\begin{array}{l}
n \\
p
\end{array}\right)} \sum_{|L|=n-p} \frac{\Omega_{L \bar{L}}}{\beta_{L}}
$$

which clearly holds since $\Omega_{L \bar{L}} \geq 0$ and $\beta_{K}>0$ for all multi-indices $K, L$.

Let us now prove (82). With the above notation, we have at $x$ :

$$
\frac{\alpha^{n-1} \wedge \beta}{\alpha^{n}}=\frac{1}{n} \sum_{j=1}^{n} \beta_{j} \quad \text { and } \quad \frac{\alpha^{p-1}}{(p-1) !}=\sum_{|J|=p-1} i d z_{J} \wedge d \bar{z}_{J}
$$

and the second identity yields at $x$ :

$$
\frac{\alpha^{p-1} \wedge \beta}{(p-1) !}=\sum_{|J|=p-1} \sum_{j \in\{1, \ldots, n\} \backslash J} \beta_{j} i d z_{j} \wedge d \bar{z}_{j} \wedge i d z_{J} \wedge d \bar{z}_{J},
$$

which, in turn, implies the following identity at $x$ :

$$
\frac{\alpha^{p-1} \wedge \beta \wedge \Omega^{n-p, n-p}}{(p-1) !(n-p) !}=\sum_{|J|=p-1} \sum_{j \in\{1, \ldots, n\} \backslash J} \beta_{j} \Omega_{L_{j J} \bar{L}_{j J}} \frac{\alpha^{n}}{n !},
$$

where we have set $\Omega_{L_{j J} \bar{L}_{j J}}:=\Omega_{L \bar{L}}$ with $L:=\{1, \ldots, n\} \backslash(\{j\} \cup J)$ ordered increasingly. Thus, $\{j\}, J$ and $L$ form a partition of $\{1, \ldots, n\}$, so any two of them uniquely determine the third.

Consequently, inequality (82) at $x$ translates to 


$$
\frac{n}{p} \frac{1}{\left(\begin{array}{l}
n \\
p
\end{array}\right) n}\left(\sum_{|L|=n-p} \Omega_{L \bar{L}}\right)\left(\sum_{j=1}^{n} \beta_{j}\right) \geq \frac{(p-1) !(n-p) !}{n !} \sum_{|J|=p-1} \sum_{j \in\{1, \ldots, n\} \backslash J} \beta_{j} \Omega_{L_{j J} \bar{L}_{j J}},
$$

which is clear since $\frac{n}{p} \frac{1}{\left(\begin{array}{c}n \\ p\end{array}\right) n}=\frac{(p-1) !(n-p) !}{n !}, \Omega_{L \bar{L}} \geq 0$ for every $L, \beta_{j}>0$ for every $j$ and the terms in the double sum on the r.h.s. of the above inequality are precisely all the products of the shape $\Omega_{L \bar{L}} \beta_{j}$ with $j \notin L$, so they form a subset of the terms on the l.h.s.

Note that inequalities (81) and (82) of Lemma 7.1 allow a kind of "simplification" of $\alpha^{n}$ between the numerators and the denominators. For possible future use, we notice a simultaneous reinforcement of inequalities (81) and (82) that has not been used in this paper. For this reason and since the proof of the general case involves rather lengthy calculations, we will only prove a special case.

Lemma 7.2 Let $\alpha, \beta$ be arbitrary Hermitian metrics on a complex manifold $X$ with dim $_{\mathbb{C}} X=n$. Let $p \in\{1, \ldots, n\}$ be arbitrary.

If $\Omega^{n-p, n-p}$ is proportional to $\alpha^{k} \wedge \beta^{n-p-k}$ for some $k \in\{0, \ldots, n-p\}$, then the factor $\left(\begin{array}{l}n \\ p\end{array}\right)$ can be omitted from (81). In other words, for all $p, k \in\{0, \ldots, n\}$ such that $p+k \leq n$ we have:

$$
\frac{\alpha^{n-p} \wedge \beta^{p}}{\alpha^{n}} \cdot \frac{\alpha^{p+k} \wedge \beta^{n-p-k}}{\alpha^{n}} \geq \frac{\alpha^{k} \wedge \beta^{n-k}}{\alpha^{n}} \text { on } X .
$$

Proof. We will only prove here the case when $p+k=n-1$, i.e.

$$
\frac{\alpha^{n-p} \wedge \beta^{p}}{\alpha^{n}} \cdot \frac{\beta \wedge \alpha^{n-1}}{\alpha^{n}} \geq \frac{\alpha^{n-p-1} \wedge \beta^{p+1}}{\alpha^{n}} \text { on } X,
$$

which is equivalent to $\left(\Lambda_{\gamma} \alpha\right)\left(\Lambda_{\alpha} \beta\right) \geq n \Lambda_{\gamma} \beta$ when $\gamma^{n-1}=t \alpha^{n-p-1} \wedge \beta^{p}$ for some constant $t>0$. Notice that this last inequality improves by a factor $n$ in the special case when $\gamma^{n-1}=t \alpha^{n-p-1} \wedge \beta^{p}$ the general lower bound proved in [Pop14, Lemma 3.1].

We fix an arbitrary point $x \in X$ and choose local coordinates as in (83). Using identities analogous to those in the proof of Lemma 7.1, we see that (86) translates at $x$ to

$$
\frac{n-p}{n}\left(\sum_{j_{1}<\cdots<j_{p}} \beta_{j_{1}} \ldots \beta_{j_{p}}\right)\left(\sum_{l=1}^{n} \beta_{l}\right) \geq(p+1) \sum_{k_{1}<\cdots<k_{p+1}} \beta_{k_{1}} \ldots \beta_{k_{p+1}}
$$

Now, the 1.h.s. of inequality (87) equals

$$
\frac{n-p}{n}\left((p+1) \sum_{k_{1}<\cdots<k_{p+1}} \beta_{k_{1}} \ldots \beta_{k_{p+1}}+\sum_{j_{1}<\cdots<j_{p}} \beta_{j_{1}} \ldots \beta_{j_{p}}\left(\beta_{j_{1}}+\cdots+\beta_{j_{p}}\right)\right),
$$

so $(87)$ is equivalent to

$$
(n-p) \sum_{j_{1}<\cdots<j_{p}} \beta_{j_{1}} \ldots \beta_{j_{p}}\left(\beta_{j_{1}}+\cdots+\beta_{j_{p}}\right) \geq p(p+1) \sum_{k_{1}<\cdots<k_{p+1}} \beta_{k_{1}} \ldots \beta_{k_{p+1}} .
$$

We will now prove (88). Let us fix an arbitrary ordered sequence $1 \leq k_{1}<\cdots<k_{p+1} \leq n$. For every $r, s \in\left\{k_{1}, \ldots, k_{p+1}\right\}$ with $r<s$, we have: 


$$
2 \beta_{k_{1}} \ldots \beta_{k_{p+1}}=\left(2 \beta_{r} \beta_{s}\right) \prod_{l \notin\{r, s\}} \beta_{l} \leq \beta_{r}^{2} \prod_{l \notin\{r, s\}} \beta_{l}+\beta_{s}^{2} \prod_{l \notin\{r, s\}} \beta_{l},
$$

where all the products above bear on the indices $l \in\left\{k_{1}, \ldots, k_{p+1}\right\} \backslash\{r, s\}$. Note that $\beta_{r}^{2} \prod_{l \notin\{r, s\}} \beta_{l}$ is obtained from $\beta_{k_{1}} \ldots \beta_{k_{p+1}}$ by omitting $\beta_{s}$ and counting $\beta_{r}$ twice. Summing up these inequalities over all the $\left(\begin{array}{c}p+1 \\ 2\end{array}\right)$ pairs of indices $r<s$ selected from $k_{1}, \ldots, k_{p+1}$, we get

$$
\begin{aligned}
\left(\begin{array}{c}
p+1 \\
2
\end{array}\right) 2 \beta_{k_{1}} \ldots \beta_{k_{p+1}} & \leq \beta_{k_{2}} \ldots \beta_{k_{p+1}}\left(\beta_{k_{2}}+\cdots+\beta_{k_{p+1}}\right) \\
& +\beta_{k_{1}} \beta_{k_{3}} \ldots \beta_{k_{p+1}}\left(\beta_{k_{1}}+\beta_{k_{3}}+\cdots+\beta_{k_{p+1}}\right) \\
& +\ldots \ldots \ldots \\
& +\beta_{k_{1}} \ldots \beta_{k_{p}}\left(\beta_{k_{1}}+\cdots+\beta_{k_{p}}\right) .
\end{aligned}
$$

Note that for every $s \in\{1, \ldots, p+1\}, \beta_{k_{s}}$ does not feature in the $s^{t h}$ line on the r.h.s. of (90). Adding up these inequalities over all the ordered sequences $1 \leq k_{1}<\cdots<k_{p+1} \leq n$, we get the desired inequality (88) because any ordered sequence $1 \leq j_{1}<\cdots<j_{p} \leq n$ occurs inside exactly $(n-p)$ ordered sequences $1 \leq k_{1}<\cdots<k_{p+1} \leq n$. Indeed, the extra index for $1 \leq k_{1}<\cdots<k_{p+1} \leq n$ can be chosen arbitrarily in $\{1, \ldots, n\} \backslash\left\{j_{1}, \ldots, j_{p}\right\}$, so there are $(n-p)$ choices for it.

This completes the proof of (88), hence the proof of (85) when $p+k=n-1$.

Again for the record, we notice that an application of Lemma 7.2 is an inequality between intersection numbers of cohomology classes reminiscent of the Hovanskii-Teissier inequalities (cf. e.g. [Dem93, Proposition 5.2]). It has an interest of its own.

Proposition 7.3 Let $X$ be a compact Kähler manifold with $\operatorname{dim}_{\mathbb{C}} X=n$ and let $\{\alpha\},\{\beta\} \in H_{B C}^{1,1}(X, \mathbb{R})$ be nef Bott-Chern cohomology classes. Then

$$
\left(\{\alpha\}^{n-p} \cdot\{\beta\}^{p}\right)\left(\{\alpha\}^{p+k} \cdot\{\beta\}^{n-p-k}\right) \geq\left(\{\alpha\}^{n}\right)\left(\{\alpha\}^{k} \cdot\{\beta\}^{n-k}\right)
$$

for all $p, k \in\{0, \ldots, n\}$ such that $p+k \leq n$.

By the density of the nef cone in the Kähler cone, we may assume without loss of generality that $\{\alpha\}$ and $\{\beta\}$ are Kähler classes in which we fix respective Kähler metrics $\alpha, \beta$.

Proof 1 (deduced from a known result). ${ }^{1}$ For every $j \in\{0, \ldots n\}$, let

$$
c_{j}:=\log \left(\{\alpha\}^{j} \cdot\{\beta\}^{n-j}\right) .
$$

It is a standard result that the function $j \mapsto c_{j}$ is concave. Now, $k \leq n-p \leq n$ and

$$
n-p=\frac{p}{n-k} k+\frac{n-k-p}{n-k} n, \quad \text { hence, by concavity, } \quad c_{n-p} \geq \frac{p}{n-k} c_{k}+\frac{n-k-p}{n-k} c_{n} .
$$

\footnotetext{
${ }^{1}$ This argument was kindly pointed out to the author by S. Boucksom
} 
Similarly, $k \leq p+k \leq n$ and

$$
p+k=\frac{n-p-k}{n-k} k+\frac{p}{n-k} n, \quad \text { hence, by concavity, } \quad c_{p+k} \geq \frac{n-p-k}{n-k} c_{k}+\frac{p}{n-k} c_{n} .
$$

Taking the sum of (92) and (93), we get: $c_{n-p}+c_{p+k} \geq c_{n}+c_{k}$, which is nothing but (91).

Proof 2. It uses the pointwise inequality (85) via the technique introduced in [Pop14] and the approximate fixed point technique introduced in the proof of Proposition 5.1. The arguments are essentially a repetition of some of those used above, so we will only indicate the main points.

First notice that the case when $k=0$ is an immediate consequence of the Hovanskii-Teissier inequalities (cf. [Dem93, Proposition 5.2]) which spell:

$$
\{\alpha\}^{n-p} \cdot\{\beta\}^{p} \geq\left(\{\alpha\}^{n}\right)^{\frac{n-p}{n}}\left(\{\beta\}^{n}\right)^{\frac{p}{n}} \text { and }\{\alpha\}^{p} \cdot\{\beta\}^{n-p} \geq\left(\{\alpha\}^{n}\right)^{\frac{p}{n}}\left(\{\beta\}^{n}\right)^{\frac{n-p}{n}} .
$$

Multiplying these two inequalities, we get (91) for $k=0$.

For the general case of an arbitrary $k$, we consider the Monge-Ampère equation:

$$
\widetilde{\alpha}^{n}=\frac{\{\alpha\}^{n}}{\{\alpha\}^{k} \cdot\{\beta\}^{n-k}} \alpha^{k} \wedge \beta^{n-k}, \quad \text { or equivalently } \quad \operatorname{det}_{\beta} \widetilde{\alpha}=\frac{\{\alpha\}^{n}}{\{\alpha\}^{k} \cdot\{\beta\}^{n-k}} \frac{\alpha^{k} \wedge \beta^{n-k}}{\beta^{n}},
$$

for which the approximate fixed point technique introduced in the proof of Proposition 5.1 produces, for every $\varepsilon>0$, a Kähler metric $\widetilde{\alpha}_{\varepsilon}$ in the Kähler class $\{\alpha\}$ (in which we have fixed beforehand a Kähler metric $\omega$ ) such that

$$
\widetilde{\alpha}_{\varepsilon}^{n}=\frac{\{\alpha\}^{n}}{\{\alpha\}^{k} \cdot\{\beta\}^{n-k}}\left[(1-\varepsilon) \omega_{\varepsilon}+\varepsilon \omega\right]^{k} \wedge \beta^{n-k} \geq(1-\varepsilon)^{k} \frac{\{\alpha\}^{n}}{\{\alpha\}^{k} \cdot\{\beta\}^{n-k}} \widetilde{\alpha}_{\varepsilon}^{k} \wedge \beta^{n-k}-O\left(\left|\eta_{\varepsilon}\right|\right),
$$

for some constant $\eta_{\varepsilon} \rightarrow 0$ as $\varepsilon \rightarrow 0$. Hence

$$
\operatorname{det}_{\beta} \widetilde{\alpha}_{\varepsilon} \geq(1-\varepsilon)^{k} \frac{\{\alpha\}^{n}}{\{\alpha\}^{k} \cdot\{\beta\}^{n-k}} \frac{\widetilde{\alpha}_{\varepsilon}^{k} \wedge \beta^{n-k}}{\beta^{n}}-O\left(\left|\eta_{\varepsilon}\right|\right) .
$$

We can now rerun the argument used several times above. For every $\varepsilon>0$, we have:

$$
\begin{aligned}
&\left(\{\alpha\}^{n-p} \cdot\{\beta\}^{p}\right)\left(\{\alpha\}^{p+k} \cdot\{\beta\}^{n-p-k}\right)=\left(\int_{X} \frac{\widetilde{\alpha}_{\varepsilon}^{n-p} \wedge \beta^{p}}{\beta^{n}} \beta^{n}\right)\left(\int_{X} \frac{\widetilde{\alpha}_{\varepsilon}^{p+k} \wedge \beta^{n-p-k}}{\widetilde{\alpha}_{\varepsilon}^{n}}\left(\operatorname{det}_{\beta} \widetilde{\alpha}_{\varepsilon}\right) \beta^{n}\right) \\
& \stackrel{(a)}{\geq}\left[\int_{X}\left(\frac{\widetilde{\alpha}_{\varepsilon}^{n-p} \wedge \beta^{p}}{\beta^{n}} \frac{\widetilde{\alpha}_{\varepsilon}^{p+k} \wedge \beta^{n-p-k}}{\widetilde{\alpha}_{\varepsilon}^{n}}\right)^{\frac{1}{2}}\left(\operatorname{det}_{\beta} \widetilde{\alpha}_{\varepsilon}\right)^{\frac{1}{2}} \beta^{n}\right]^{2} \\
& \stackrel{(b)}{\geq}\left[\int_{X}\left(\frac{\widetilde{\alpha}_{\varepsilon}^{k} \wedge \beta^{n-k}}{\beta^{n}}\right)^{\frac{1}{2}}\left(\operatorname{det}_{\beta} \widetilde{\alpha}_{\varepsilon}\right)^{\frac{1}{2}} \beta^{n}\right]^{2} \\
& \stackrel{(c)}{\geq}(1-\varepsilon)^{k} \frac{\{\alpha\}^{n}}{\{\alpha\}^{k} \cdot\{\beta\}^{n-k}}\left(\int_{X} \widetilde{\alpha}_{\varepsilon}^{k} \wedge \beta^{n-k}\right)^{2}-O\left(\left|\eta_{\varepsilon}\right|\right) \\
&=(1-\varepsilon)^{k}\{\alpha\}^{n}\left(\{\alpha\}^{k} \cdot\{\beta\}^{n-k}\right)-O\left(\left|\eta_{\varepsilon}\right|\right) .
\end{aligned}
$$


As usual, (a) follows from the Cauchy-Schwarz inequality, (b) follows from the pointwise inequality (85), while $(c)$ follows from the inequality (95). Letting $\varepsilon \rightarrow 0$, we get (91).

\section{References.}

[AB93] L. Alessandrini, G. Bassanelli - Metric Properties of Manifolds Bimeromorphic to Compact Kähler Spaces — J. Diff. Geom. 37 (1993), 95-121.

[AB96] L. Alessandrini, G. Bassanelli — The class of Compact Balanced Manifolds Is Invariant under Modifications - Complex Analysis and Geometry (Trento, 1993), 117, Lecture Notes in Pure and Appl. Math., 173, Dekker, New York, 1996.

[BT82] E. Bedford, B. Taylor — A New Capacity for Plurisubharmonic Functions. — Acta Math. 149 (1982), 140.

[BK07] Z. Blocki, S. Kolodziej — On Regularization of Plurisubharmonic Functions on Manifolds - Proc. AMS 135, no. 7, (2007), 2089-2093.

[Bou02] S. Boucksom - On the Volume of a Line Bundle - Internat. J. Math. 13 (2002), no. 10, 10431063.

[BDPP13] S. Boucksom, J.-P. Demailly, M. Paun, T. Peternell - The Pseudo-effective Cone of a Compact Kähler Manifold and Varieties of Negative Kodaira Dimension - J. Alg. Geom. 22 (2013) 201-248.

[BEGZ10] S. Boucksom, P. Eyssidieux, V. Guedj, A. Zeriahi - Monge-Ampère Equations in Big Cohomology Classes — Acta Math. 205 (2010), 199-262.

[BFJ09] S. Boucksom, C. Favre, M. Jonsson - Differentiability of Volumes of Divisors and a Problem of Teissier — J. Alg. Geom 18 (2009) 279-308.

[CRS14] I. Chiose, R. Rasdeaconu, I.Suvaina - Balanced Metrics on Uniruled Manifolds - arXiv e-print DG 1408.4769v1.

[Dem85] J.-P. Demailly — Mesures de Monge-Ampère et caractérisation géométrique des variétés algébriques affines — Mém. Soc. Math. France (N.S.) 19 (1985) 1-124.

[Dem92] J.-P. Demailly — Regularization of Closed Positive Currents and Intersection Theory - J. Alg. Geom., 1 (1992), 361-409.

[Dem93] J.-P. Demailly - A Numerical Criterion for Very Ample Line Bundles - J. Diff. Geom. 37 (1993) 323-374.

[Dem 97] J.-P. Demailly — Complex Analytic and Algebraic Geometry -http://www-fourier.ujfgrenoble.fr/ demailly/books.html

[DP04] J.-P. Demailly, M. Paun - Numerical Characterization of the Kähler Cone of a Compact Kähler Manifold - Ann. of Math. 159 (2004), 1247-1274.

[FLM11] H. Fang, M. Lai, X. Ma - On a Class of Fully Nonlinear Flows in Kähler Geometry J. reine angew. Math. 653 (2011), 189220. 
[Gau77] P. Gauduchon - Le théorème de l'excentricité nulle - C.R. Acad. Sc. Paris, Série A, t. 285 (1977), 387-390.

[GZ05] V. Guedj, A. Zeriahi — Intrinsic Capacities on Compact Kähler Manifolds - J. Geom. Anal. 15 (2005), no. 4, 607639.

[Lam99] A. Lamari - Courants kählériens et surfaces compactes — Ann. Inst. Fourier, Grenoble, 49, 1 (1999), 263-285.

[Pop13] D. Popovici - Aeppli Cohomology Classes Associated with Gauduchon Metrics on Compact Complex Manifolds - arXiv e-print DG 1310.3685v1, to appear in Bull. Soc. Math. France.

[Pop14] D. Popovici - Sufficient Bigness Criterion for Differences of Two Nef Classes - Math. Ann. DOI 10.1007/s00208-015-1230-z (new title of An Observation Relative to a Paper by J. Xiao - arXiv e-print DG 1405.2518v1).

[Siu74] Y.-T. Siu - Analyticity of Sets Associated to Lelong Numbers and the Extension of Closed Positive Currents - Invent. Math. 27 (1974), 53-156.

[Tom10] M. Toma - A Note on the Cone of Mobile Curves - C. R. Math. Acad. Sci. Paris 348 (2010), 7173.

[Tos15] V. Tosatti — The Calabi-Yau Theorem and Kähler Currents — arXiv 1505.02124.

[TW10] V. Tosatti, B. Weinkove - The Complex Monge-Ampère Equation on Compact Hermitian Manifolds - J. Amer. Math. Soc. 23 (2010), no. 4, 1187-1195.

[Xia13] J. Xiao - Weak Transcendental Holomorphic Morse Inequalities on Compact Kähler Manifolds - to appear in Ann. Inst. Fourier.

[Yau78] S.T. Yau - On the Ricci Curvature of a Complex Kähler Manifold and the Complex MongeAmpère Equation — Comm. Pure Appl. Math. 31 (1978) 339-411.

Institut de Mathématiques de Toulouse, Université Paul Sabatier, 118 route de Narbonne, 31062 Toulouse, France

Email: popovici@math.univ-toulouse.fr 